\title{
CLASSIFICATION OF BASES OF TWISTED AFFINE ROOT SUPERSYSTEMS
}

\author{
Malihe Yousofzadeh[1
}

\begin{abstract}
Following the definition of a root basis of an affine root system, we define a base of the root system of an affine Lie superalgebra to be a linearly independent subset $B$ of its root system such that each root can be written as a linear combination of elements of $B$ with integral coefficients such that all coefficients are nonnegative or all coefficients are nonpositive. Characterization and classification of bases of root systems of affine Lie algebras are known in the literature; in fact, up to \pm 1 -multiple, each base of an affine root system is conjugate with the standard base under the Weyl group action. In the super case, the existence of those self-orthogonal roots which are not orthogonal to at least one other root, makes the situation more complicated. In this work, we give a complete characterization of bases of a twisted affine root supersystem. We precisely describe and classify them.
\end{abstract}

\section{INTRODUCTION}

The concept of a base is crucial to study highest weight modules of finite dimensional basic classical simple Lie superalgebras and affine Lie (super)algebras. The purpose of this work is finding a characterization and giving the classification of bases of the root systems of twisted affine Lie superalgebras; affine Lie superalgebras are the super version of affine Lie algebras which have been defined, constructed and classified by J.W. Van de Lour [8] in 1986. In what follows we briefly recall their structure and classification:

Suppose that $n$ is a positive integer and $I=\{1, \ldots, n\}$. Let $\tau$ be a subset of $I$ and $A$ be a nonzero indecomposable symmetrizable $n \times n$-matrix with complex entries (in which by symmetrizable, we mean that $A$ has a decomposition $A=D B$ with an invertible diagonal matrix $D$ and a symmetric matrix $B$ ), satisfying the

2010 Mathematics Subject Classification: 17B67.

Key Words: Twisted Affine Lie Superalgebra; Affine root supersystem; Base; Quasi-Weyl group.

${ }^{1}$ Department of Mathematics, University of Isfahan, Isfahan, Iran, P.O.Box 81745-163, and School of Mathematics, Institute for Research in Fundamental Sciences (IPM), P.O. Box: 193955746, Tehran, Iran. Email address: ma.yousofzadeh@sci.ui.ac.ir \& ma.yousofzadeh@ipm.ir. This research was in part supported by a grant from IPM (No. 98170424) and is partially carried out in IPM-Isfahan Branch. 
following:

$$
\begin{aligned}
& \text { if } a_{i, j}=0, \quad \text { then } a_{j, i}=0 \\
& \text { if } a_{i, i}=0, \quad \text { then } i \in \tau ; \\
& \text { if } a_{i, i} \neq 0, \quad \text { then } a_{i, i}=2 ; \\
& \text { if } a_{i, i} \neq 0, \quad \text { then } a_{i, j}\left(\text { resp. } a_{i, j} / 2\right) \text { is a nonpositive integer } \\
& \quad \text { for } i \in I \backslash \tau(\text { resp. } i \in \tau) \text { with } i \neq j .
\end{aligned}
$$

Fix a complex vector space $\mathcal{H}$ of dimension $n+\operatorname{corank}(A)$ and denote its dual space with $\mathcal{H}^{*}$. Then there exist linearly independent subsets

$$
\Pi:=\left\{\alpha_{i} \mid i \in I\right\} \subseteq \mathcal{H}^{*} \text { and } \check{\Pi}:=\left\{\check{\alpha}_{i} \mid i \in I\right\} \subseteq \mathcal{H}
$$

such that

$$
\alpha_{j}\left(\check{\alpha}_{i}\right)=a_{i, j} \quad(i, j \in I) .
$$

Let $\widetilde{\mathcal{G}}(A, \tau)$ be the Lie superalgebra generated by $\left\{e_{i}, f_{i} \mid i \in I\right\} \cup \mathcal{H}$ subject to the following relations:

$$
\begin{array}{ll}
{\left[e_{i}, f_{j}\right]=\delta_{i, j} \check{\alpha}_{i},} & {\left[h, h^{\prime}\right]=0,} \\
{\left[h, e_{j}\right]=\alpha_{j}(h) e_{j},} & {\left[h, f_{j}\right]=-\alpha_{j}(h) f_{j},} \\
\operatorname{deg}(h)=0, & \operatorname{deg}\left(e_{i}\right)=\operatorname{deg}\left(f_{i}\right)= \begin{cases}0 & \text { if } i \notin \tau \\
1 & \text { if } i \in \tau\end{cases}
\end{array}
$$

for $i, j \in I$ and $h, h^{\prime} \in \mathcal{H}$, where $\delta_{i, j}$ denotes the Kronecker delta. There is a unique maximal ideal $\mathfrak{i}$ of $\widetilde{\mathcal{G}}(A, \tau)$ intersecting $\mathcal{H}$ trivially. The Lie superalgebra $\mathcal{G}:=\widetilde{\mathcal{G}}(A, \tau) / \mathfrak{i}$ is called an affine Lie superalgebra if it is not of finite dimension but of finite growth; see [8, §6.1]. By a convention, the images of $e_{i}, f_{i}$ and $h$ $(i \in I, h \in \mathcal{H})$ in $\mathcal{G}(A, \tau)$ under the canonical projection map are denoted again by $e_{i}, f_{i}$ and $h$, respectively. Then $\mathcal{G}$ is generated by $\left\{e_{i}, f_{i} \mid 1 \leq i \leq n\right\} \cup \mathcal{H}$. The Lie superalgebra $\mathcal{G}$ has a root space decomposition with respect to $\mathcal{H}$. Moreover, $\Pi$ is a subset of the corresponding root system $R$ and each element of $R$ is written as a $\mathbb{Z}$-linear combination of elements of $\Pi$ whose coefficients are all nonnegative or all are nonpositive; we refer to $\Pi$ as the standard base of $R$. Elements of $R$ are called roots and roots corresponding to even and odd part of $\mathcal{G}$ are called respectively even and odd.

As for affine Lie algebras, affine Lie superalgebras are constructed using an affinization process: Suppose that $\mathfrak{g}:=\mathfrak{g}_{0} \oplus \mathfrak{g}_{1}$ is a finite dimensional basic classical simple Lie superalgebra with a Cartan subalgebra $\mathfrak{h} \subseteq \mathfrak{g}_{0}$. Suppose that $\kappa$ is a nondegenerate supersymmetric invariant even bilinear form on $\mathfrak{g}$ and $\sigma$ is an automorphism of $\mathfrak{g}$ of order $k$. Since $\sigma$ preserves $\mathfrak{g}_{0}$ as well as $\mathfrak{g}_{1}$, we have

$$
\mathfrak{g}_{i}=\bigoplus_{s=0}^{k-1}[s] \mathfrak{g}_{i} \quad \text { where } \quad[s] \mathfrak{g}_{i}=\left\{x \in \mathfrak{g}_{i} \mid \sigma(x)=\zeta^{s} x\right\} \quad\left(i \in \mathbb{Z}_{2}, 0 \leq s \leq k-1\right)
$$

in which $\zeta$ is the $k$-th primitive root of unity. Then

$$
\widehat{\mathfrak{g}}:=\widehat{\mathfrak{g}}_{0} \oplus \widehat{\mathfrak{g}}_{1} \quad \text { where } \quad \widehat{\mathfrak{g}}_{i}:=\bigoplus_{s=0}^{k-1}\left({ }^{[s]} \mathfrak{g}_{i} \otimes t^{s} \mathbb{C}\left[t^{ \pm k}\right]\right) \quad\left(i \in \mathbb{Z}_{2}\right)
$$

is a subalgebra of the current superalgebra $\mathfrak{g} \otimes \mathbb{C}\left[t^{ \pm 1}\right]$. Set

$$
\mathscr{G}=\bigoplus_{s=0}^{k-1}\left({ }^{[s]} \mathfrak{g} \otimes t^{s} \mathbb{C}\left[t^{ \pm k}\right]\right) \oplus \mathbb{C} c \oplus \mathbb{C} d \quad \text { and } \quad \mathscr{H}:=\left(\left({ }^{[0]} \mathfrak{g} \cap \mathfrak{h}\right) \otimes 1\right) \oplus \mathbb{C} c \oplus \mathbb{C} d
$$


then $\mathscr{G}$ together with

$\left[x \otimes t^{p}+r c+s d, y \otimes t^{q}+r^{\prime} c+s^{\prime} d\right]:=[x, y] \otimes t^{p+q}+p \kappa(x, y) \delta_{p+q, 0} c+s q y \otimes t^{q}-s^{\prime} p x \otimes t^{p}$

is an affine Lie superalgebra and $\mathscr{H}$ is a Cartan subalgebra of $\mathscr{G}$. The Lie superalgebra $\mathscr{G}$ is denoted by $X^{(k)}$ where $X$ is the type of $\mathfrak{g}$ and $X \neq A(\ell, \ell)$. The definition of $A(\ell, \ell)^{(1)}$ is a little bit different. The Lie superalgebra $X^{(k)}$ is called twisted if $k>1$ and it is called non-twisted if $k=1$. Twisted and non-twisted affine Lie superalgebras cover all affine Lie superalgebras. We refer to the root system $R$ of $\mathscr{G}$, an affine root supersystem.

Define $\delta$ to be the functional on $\mathscr{H}$ vanishing on $\left(\left({ }^{[0]} \mathfrak{g} \cap \mathfrak{h}\right) \otimes 1\right) \oplus \mathbb{C} c$ and mapping $d$ to 1 . If $\dot{R}$ is the root system of $\mathfrak{g}, \dot{R}+\mathbb{Z} \delta$ is the root system of $\mathscr{G}=X^{(1)}$.

Using the form $\kappa$ on $\mathfrak{g}$, one has a nondegenerate supersymmetric invariant even bilinear form on $\mathscr{G}$ which in turn, induces a symmetric bilinear form $(\cdot, \cdot)$ on the dual space $\mathscr{H}^{*}$ of $\mathscr{H}$. Moreover, if $\mathscr{G}$ is twisted, for nonnegative integers $m$ and $n$ as in the following table and $V:=\operatorname{span}_{\mathbb{R}} R$, there are $\epsilon_{i}, \delta_{p} \in V(1 \leq i \leq m, 1 \leq p \leq n)$ such that

$$
\left(\epsilon_{i}, \epsilon_{j}\right):=\delta_{i, j}, \quad\left(\delta_{p}, \delta_{q}\right):=-\delta_{p, q}, \quad\left(\epsilon_{i}, \delta_{p}\right):=0, \quad(\delta, V)=\{0\},
$$

$\mathscr{G}$ is one of the Lie superalgebras in the first column of the following table and $R$ is correspondingly expressed as follows:

TABLE 1.

\begin{tabular}{|c|rll|}
\hline Type & & \multicolumn{1}{|c|}{$R$} \\
\hline$A(2 m, 2 n-1)^{(2)}$ & $\mathbb{Z} \delta$ & $\cup$ & $\mathbb{Z} \delta \pm\left\{\epsilon_{i}, \delta_{j}, \epsilon_{i} \pm \epsilon_{r}, \delta_{j} \pm \delta_{s}, \epsilon_{i} \pm \delta_{j} \mid i \neq r, j \neq s\right\}$ \\
$(n \neq 0)$ & & $\cup$ & $(2 \mathbb{Z}+1) \delta \pm\left\{2 \epsilon_{i} \mid 1 \leq i \leq m\right\}$ \\
& & $\cup$ & $2 \mathbb{Z} \delta \pm\left\{2 \delta_{j} \mid 1 \leq j \leq n\right\}$ \\
\hline$A(2 m-1,2 n-1)^{(2)}$ & $\mathbb{Z} \delta$ & $\cup$ & $\mathbb{Z} \delta \pm\left\{\epsilon_{i} \pm \epsilon_{r}, \delta_{j} \pm \delta_{s}, \delta_{j} \pm \epsilon_{i} \mid i \neq r, j \neq s\right\}$ \\
$m, n \neq 0,(m, n) \neq(1,1)$ & & $\cup$ & $(2 \mathbb{Z}+1) \delta \pm\left\{2 \epsilon_{i} \mid 1 \leq i \leq m\right\}$ \\
& & $\cup$ & $2 \mathbb{Z} \delta \pm\left\{2 \delta_{j} \mid 1 \leq j \leq n\right\}$ \\
\hline & $\mathbb{Z} \delta$ & $\cup$ & $\mathbb{Z} \delta \pm\left\{\epsilon_{i}, \delta_{j} \mid 1 \leq i \leq m, 1 \leq j \leq n\right\}$ \\
$\left(n(2 m, 2 n)^{(4)}\right.$ & & $\cup$ & $2 \mathbb{Z} \delta \pm\left\{\epsilon_{i} \pm \epsilon_{r}, \delta_{j} \pm \delta_{s}, \delta_{j} \pm \epsilon_{i} \mid i \neq r, j \neq s\right\}$ \\
$(n, m) \neq(0,0)$ & & $\cup$ & $(4 \mathbb{Z}+2) \delta \pm\left\{2 \epsilon_{i} \mid 1 \leq i \leq m\right\}$ \\
& & $\cup$ & $\mathbb{Z} \delta \pm\left\{2 \delta_{j} \mid 1 \leq j \leq n\right\}$ \\
\hline$D(m+1, n)^{(2)}$ & $\mathbb{Z} \delta$ & $\cup$ & $\mathbb{Z} \delta \pm\left\{\epsilon_{i}, \delta_{j} \mid 1 \leq i \leq m, 1 \leq j \leq n\right\}$ \\
$(n \neq 0)$ & & $\cup$ & $2 \mathbb{Z} \delta \pm\left\{2 \delta_{j}, \epsilon_{i} \pm \epsilon_{r}, \delta_{j} \pm \delta_{s}, \delta_{j} \pm \epsilon_{i} \mid i \neq r, j \neq s\right\}$ \\
\hline
\end{tabular}

where we make a convention that if $m=0$ or $n=0$, then their corresponding expressions disappear. We set

$$
\begin{array}{lll}
R^{0}:=\mathbb{Z} \delta, & R^{\times}:=R \backslash R^{0}, & \\
R_{r e}^{\times}:=\{\alpha \in R \mid(\alpha, \alpha) \neq 0\}, & R_{r e}:=R_{r e}^{\times} \cup\{0\} & \text { (real roots), } \\
R_{n s}^{\times}:=\left\{\alpha \in R^{\times} \mid(\alpha, \alpha)=0\right\}, & R_{n s}:=R_{n s}^{\times} \cup\{0\} & \text { (nonsingular roots) }
\end{array}
$$

and mention that the existence of nonzero nonsingular roots is a phenomena which does not occur for affine Lie algebras.

We recall that the Weyl group of an affine root (super)system $R$ is the group generated by linear transformations $\sigma_{\alpha}$, for nonzero real roots $\alpha$, on $V=\operatorname{span}_{\mathbb{R}} R$ mapping $\beta \in V$ to $\beta-2 \frac{(\beta, \alpha)}{(\alpha, \alpha)} \alpha$. As all nonzero nonsingular roots are odd and two times of an odd real root is an even real root we call $\sigma_{\alpha}$ 's $\left(\alpha \in R_{r e}^{\times}\right)$even reflections.

Following the definition of a root basis of an affine root system; see [3, §5.9], we define a base of the root system $R$ of an affine Lie superalgebra $\mathscr{G}$ to be a linearly 
independent subset $B$ of $R$ such that each element of $R$ can be written as a linear combination of elements of $B$ with integral coefficients such that all coefficients are nonnegative or all coefficients are nonpositive.

Characterization and classification of bases of root systems of affine Lie algebras are known in the literature; in fact, up to \pm 1 -multiple, each base of an affine root system is conjugate with the standard base under the Weyl group action; see [3, Pro. 5.9]. The existence of nonsingular roots in the super case makes the situation more complicated.

In [6], V. Serganova defines a base ${ }^{2}$ of $R$ to be a linearly independent subset $B$ of $R$ such that for each $\alpha \in B$, there are root vectors $x_{\alpha}$ and $y_{\alpha}$ corresponding to $\alpha$ and $-\alpha$ respectively such that $\left\{x_{\alpha}, y_{\alpha} \mid \alpha \in B\right\} \cup \mathscr{H}$ generates the affine Lie superalgebra $\mathscr{G}$ and $\left[x_{\alpha}, y_{\beta}\right]=0$ for $\alpha \neq \beta$; we refer to such a base as an S-base. It is trivial that each S-base is a base in our sense.

One of the most important differences between bases and S-bases is that bases are purely combinatorial objects while the algebraic feature of an affine Lie superalgebra gets involved to define S-bases.

The author in [6] introduces "odd reflections" to get a similar result as in nonsuper case to describe S-bases; more precisely, for a nonsingular root $\alpha$ of an S-base $B$, she defines a map

$$
s_{\alpha}: B \longrightarrow R \quad \beta \mapsto\left\{\begin{array}{cl}
-\alpha & \text { if } \beta=\alpha \\
\alpha+\beta & \text { if } \beta+\alpha \in R \\
\beta & \text { otherwise }
\end{array}\right.
$$

called an odd reflection and shows that for an S-base $B$ and a nonsingular root $\alpha \in B, s_{\alpha}(B)$ is also an S-base of $R$, moreover, up to \pm 1 -multiple, each S-base of $R$ is obtained from $B$ by iterating the action of odd and even reflections 6 , Thm. 8.3 \& Lem. 2.4].

In contrast to an even reflection which depends only on a nonzero real root, an odd reflection based on a nonzero nonsingular root, depends also on an S-base to which the root belongs., i.e., to get all S-bases, one needs to choose an S-base $\Pi$ and form all S-bases obtained from $\Pi$ by even reflections as well as odd reflections based on nonsingular roots of $\Pi$ and then form all obtained S-bases from each of these new S-bases and continue this process.

In this work, we give a complete characterization of bases of a twisted affine root supersystem $R$. We precisely describe and classify them. As a by-product, we show that for twisted affine root supersystems, there is no difference between bases and S-bases.

To have a better view of what we have in this paper, let us state our main theorem: Suppose that $R$ is a twisted affine root supersystem (see Table 11) and keep the same notation as above. We define

$$
\begin{aligned}
(\cdot, \cdot)_{*}: V \times V & \longrightarrow \mathbb{C} \\
(\delta, V)_{*} & :=\{0\},\left(\epsilon_{i}, \epsilon_{j}\right)_{*}:=\delta_{i, j},\left(\delta_{p}, \delta_{q}\right)_{*}:=\delta_{p, q} \text { and }\left(\epsilon_{i}, \delta_{p}\right)_{*}=0
\end{aligned}
$$

for $1 \leq i, j \leq m$ and $1 \leq p, q \leq n$. For $\alpha \in R$ with $(\alpha, \alpha)_{*} \neq 0$, the quasi-reflection $r_{\alpha}$ (based on $\alpha$ ) is defined to be the linear automorphism of $V$ mapping $a \in V$ to $a-2 \frac{(a, \alpha)_{*}}{(\alpha, \alpha)_{*}} \alpha$. The subgroup $W$ of the group of automorphisms of $V$ generated by $\left\{r_{\alpha} \mid \alpha \in R ;(\alpha, \alpha)_{*} \neq 0\right\}$ is called the quasi-Weyl group of $R$.

\footnotetext{
${ }^{2}$ She defines a base for all Kac-Moody Lie superalgebras in general.
} 
For $v=\sum_{i=1}^{m} t_{\epsilon_{i}} \epsilon_{i}+\sum_{p=1}^{n} t_{\delta_{p}} \delta_{p}+m \delta \in V$, we define the support of $v$ to be

$$
\operatorname{supp}(v):=\left\{\epsilon_{i} \mid 1 \leq i \leq m, t_{\epsilon_{i}} \neq 0\right\} \cup\left\{\delta_{p} \mid 1 \leq p \leq n, t_{\delta_{p}} \neq 0\right\} .
$$

For $\zeta \in \operatorname{supp}(v)$, we set

$$
\operatorname{sgn}(\zeta ; v)= \begin{cases}1 & \text { if } t_{\zeta}>0 \\ -1 & \text { if } t_{\zeta}<0\end{cases}
$$

and for $S \subseteq V$, we set

$$
\operatorname{supp}(S):=\bigcup_{\alpha \in S} \operatorname{supp}(\alpha)
$$

Theorem 1.1 (Main Theorem). Set $\ell:=m+n$. Assume $k_{1}, \ldots, k_{\ell} \in \mathbb{Z}$ and $\zeta_{1}, \ldots \zeta_{\ell} \in\left\{ \pm \epsilon_{i}, \pm \delta_{p} \mid 1 \leq i \leq m, 1 \leq p \leq n\right\}$ with $\zeta_{i} \neq \pm \zeta_{j}$ for $i \neq j$. Set

$$
\theta_{i}:=\zeta_{i}+k_{i} \delta \quad(1 \leq i \leq \ell)
$$

\begin{tabular}{|c|c|c|}
\hline$R$ & $\Pi$ & Further Assumptions \\
\hline$A(2 m, 2 n)^{(4)}$ & $\left\{\theta_{i}-\theta_{i+1}, \theta_{\ell}, \delta-\theta_{1}\right\}_{i=1}^{\ell-1}$ & $k_{i} \equiv k_{j}(\bmod 2)$ \\
\hline$D(m+1, n)^{(2)}$ & $\left\{\theta_{i}-\theta_{i+1}, \theta_{\ell}, \delta-\theta_{1}\right\}_{i=1}^{\ell-1}$ & $k_{i} \equiv k_{j}(\bmod 2)$ \\
\hline$A(2 m, 2 n-1)^{(2)}$ & $\begin{array}{l}\left\{-2 \theta_{1}+\delta, \theta_{i}-\theta_{i+1}, \theta_{\ell}\right\}_{i=1}^{\ell-1} \\
\left\{\delta-\left(\theta_{1}+\theta_{2}\right), \theta_{i}-\theta_{i+1}, \theta_{\ell}\right\}_{i=1}^{\ell-1}\end{array}$ & $\begin{array}{l}\operatorname{supp}\left(\theta_{1}\right) \subseteq\left\{\epsilon_{i} \mid 1 \leq i \leq m\right\} \\
\overline{\operatorname{supp}} \overline{\left(\theta_{1}\right)} \subseteq\left\{\bar{\delta}_{p} \mid \overline{1} \leq \bar{p}-\overline{\leq} \bar{T}\right\}\end{array}$ \\
\hline $\begin{array}{c}A(2 m-1,2 n-1)^{(2)} \\
\quad(m, n) \neq(1,1)\end{array}$ & $\begin{array}{l}\left\{\delta-\left(\theta_{1}+\theta_{2}\right), \theta_{i}-\theta_{i+1}, \theta_{\ell-1}+\theta_{\ell}\right\}_{i=1}^{\ell-1} \\
------------------- \\
\left\{-2 \theta_{1}, \theta_{i}-\theta_{i+1}, \theta_{\ell-1}+\theta_{\ell}+\delta\right\}_{i=1}^{\ell-1} \\
-------------------- \\
\left\{-2 \theta_{1}+\delta, \theta_{i}-\theta_{i+1}, \theta_{\ell-1}+\theta_{\ell}\right\}_{i=1}^{\ell-1} \\
--------------------- \\
\left\{-2 \theta_{1}, \theta_{i}-\theta_{i+1}, 2 \theta_{\ell}+\delta\right\}_{i=1}^{\ell-1}\end{array}$ & 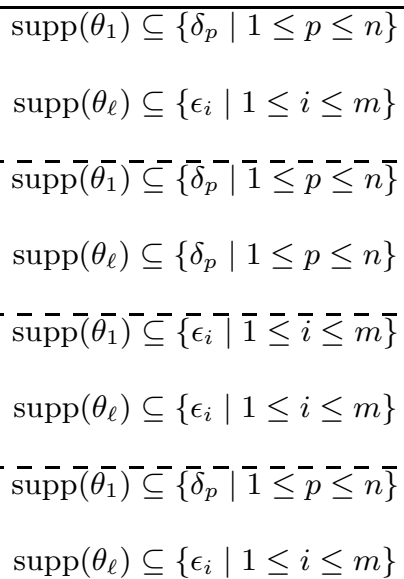 \\
\hline
\end{tabular}

and assume $\Pi$ is as in the following table:

TABLE 2.

Then $\Pi$ is a base of $R$ and each base of $R$ is of the form $\pm \Pi$. In particular, each base of $R$ is an S-base. Moreover, for each type, bases in different rows are not conjugate under quasi-Weyl group $W$ of $R$ and bases of the same form of each row are conjugate under $W$. 


\section{BASES OF TWISTED AFFINE ROOT SUPERSYSTEMS}

Suppose $\mathscr{G}$ is a twisted affine Lie superalgebra with Cartan subalgebra $\mathscr{H}$ and root system $R$; keep the same notation as in Introduction, recall the form $(\cdot, \cdot), \epsilon_{i}$ 's, $\delta_{p}$ 's, Table 1 and that $V=\operatorname{span}_{\mathbb{R}} R$.

Definition 2.1. $\quad$ (i) A linearly independent subset of $R$ is called a base of $R$ if each element of $R$ can be written as a linear combination of elements of $\Pi$ with integeral coefficients such that all coefficients are nonnegative or all coefficients are nonpositive. The set of nonzero roots of $R \cap \operatorname{span}_{\mathbb{Z} \geq 0} \Pi$ (resp. $R \cap \operatorname{span}_{\mathbb{Z} \leq 0} \Pi$ ) is denoted by $R^{+}(\Pi)$ (resp. $R^{-}(\Pi)$ ) and their elements are called positive (resp. negative) roots.

(ii) A linearly independent subset $\Pi$ of $R$ is called an S-base if for each $\alpha \in \Pi$, there are nonzero root vectors $x_{\alpha}$ and $y_{\alpha}$ corresponding to $\alpha$ and $-\alpha$ respectively such that $\left\{x_{\alpha}, y_{\alpha} \mid \alpha \in \Pi\right\} \cup \mathscr{H}$ generates the affine Lie superalgebra $\mathscr{G}$ and $\left[x_{\alpha}, y_{\beta}\right]=0$ for $\alpha \neq \beta$.

Lemma 2.2. Suppose that $\Pi$ is a base of $R$. Then $\Pi \cap 2 R=\Pi \cap \mathbb{Z} \delta=\emptyset$.

Proof. If $\alpha, 2 \alpha \in R$ and $2 \alpha \in \Pi$, then $\alpha \in \frac{1}{2} \Pi$ which is a contradiction. Also if $k \delta \in \Pi$ for some $k \in \mathbb{Z}$, then for $\alpha \in \Pi \backslash \mathbb{Z} \delta, \alpha-4 k \delta \in R$ has been written as a $\mathbb{Z}$-linear combination of the elements of $\Pi$ with the opposite signs which is a contradiction.

Lemma 2.3. $\quad$ (i) Each $S$-base of $R$ is a base of $R$.

(ii) Suppose that $\sigma$ is an even reflection. If $\Pi$ is an $S$-base of $R$, then $\sigma(\Pi)$ is also an $S$-base of $R$.

(iii) Suppose that $\Pi$ is an $S$-base of $R$ and $\sigma$ is an odd reflection based on a nonsingular root of $\Pi$. Then $\sigma(\Pi)$ is also an $S$-base of $R$.

(iv) Suppose that $\Pi$ is an $S$-base of $R$. If $B$ is a base of $R$ such that either $R^{+}(\Pi) \cap R^{-}(B)$ or $R^{+}(\Pi) \cap R^{+}(B)$ is a finite set, then $B$ is an $S$-base.

Proof. (i) follows from the definition.

(ii) Suppose that $\alpha$ is a nonzero real root. Replacing $\alpha$ with $2 \alpha$ if it is necessary, we assume $\alpha$ is an even real root. Fix $e_{\alpha} \in \mathscr{G}^{\alpha}$ and $f_{\alpha} \in \mathscr{G}^{-\alpha}$ such that $\left(e_{\alpha},\left[e_{\alpha}, f_{\alpha}\right], f_{\alpha}\right)$ is an $\mathfrak{s l}_{2}$-triple. Define

$$
\theta_{\alpha}:=\exp \left(\operatorname{ad}_{e_{\alpha}}\right) \exp \left(-\operatorname{ad}_{f_{\alpha}}\right) \exp \left(\operatorname{ad}_{e_{\alpha}}\right) .
$$

Then $\theta_{\alpha}$ ia an automorphism of $\mathscr{G}$ and we have

$$
\theta_{\alpha}(\mathcal{H})=\mathcal{H} \quad \text { and } \quad \theta_{\alpha}\left(\mathscr{G}^{\beta}\right)=\mathscr{G}^{r_{\alpha}(\beta)} \quad\left(\beta \in R_{r e}^{\times} \cup R_{n s}^{\times}\right) .
$$

Assume $\Pi$ is an S-base. Using part (i) and Lemma 2.2 , we have $\Pi \subseteq R_{r e}^{\times} \cup R_{n s}^{\times}$. Fix weight vectors $x_{\beta} \in \mathscr{G}^{\beta}$ and $y_{\beta} \in \mathscr{G}^{-\beta}(\beta \in \Pi)$ such that $\left[x_{\beta}, y_{\gamma}\right]=0$ for $\beta \neq \gamma$ and $\left\{x_{\beta}, y_{\beta} \mid \beta \in \Pi\right\} \cup \mathcal{H}$ generates $\mathscr{G}$. Then we have $\left[\theta_{\alpha}\left(x_{\beta}\right), \theta_{\alpha}\left(y_{\gamma}\right)\right]=0$ for $\beta \neq \gamma$ and (2.1) implies that $\left\{\theta_{\alpha}\left(x_{\beta}\right), \theta_{\alpha}\left(y_{\beta}\right) \mid \beta \in \Pi\right\} \cup \mathcal{H}$ is a generating set for $\mathscr{G}$. Since $\theta_{\alpha}\left(x_{\beta}\right) \in \mathscr{G}^{r_{\alpha}(\beta)}$ and $\theta_{\alpha}\left(y_{\beta}\right) \in \mathscr{G}^{-r_{\alpha}(\beta)}$, we are done.

(iii) See Lemma 3.1 of [6].

(iv) Using Lemma 4.4 of 6] together with parts (ii) and (iii) and the same argument as in [6, Lem. 4.5], we get that $B$ or $-B$ is obtained from $\Pi$ by even and odd reflections. Therefore parts (ii) and (iii) imply that $B$ is an S-base.

We mention that the twisted affine root supersystem $R$ is not necessarily preserved by the quasi-Weyl group of $R$; e.g., in each of the types listed in Table 1 . 
$r_{\epsilon_{i}-\delta_{p}}\left(2 \delta_{p}\right)=2 \epsilon_{i} \notin R$, for $1 \leq i \leq m$ and $1 \leq p \leq n$. But one can easily verify the following lemma.

Lemma 2.4. Suppose that $w$ is an element of the quasi-Weyl group of $R$ with $w^{-1}(R) \subseteq R$. If $\Pi$ is a base of $R$, then $w(\Pi)$ is also a base of $R$.

Corollary 2.5. Assume $R=A(2 k-1,2 \ell-1)^{(2)},(k, \ell) \neq(1,1)$ and suppose that $p, q \in \mathbb{Z}$ and $\zeta, \eta \in\left\{ \pm \epsilon_{i}, \pm \delta_{j} \mid 1 \leq i \leq m, 1 \leq j \leq n\right\}$ with $\zeta \neq \pm \eta$. Define

$$
\begin{aligned}
& \mathscr{I}_{\zeta, p}:= \begin{cases}r_{2 \zeta+p \delta} & \text { if } \zeta \in\left\{ \pm \delta_{j} \mid 1 \leq j \leq n\right\} \text { and } p \in 2 \mathbb{Z} \delta \\
r_{2 \zeta+p \delta} & \text { if } \zeta \in\left\{ \pm \epsilon_{i} \mid 1 \leq i \leq m\right\} \text { and } p \in 2 \mathbb{Z} \delta+\delta,\end{cases} \\
& \mathscr{J}_{\zeta, \eta, p}:=r_{\zeta-\eta+p \delta} \quad \text { if } \zeta, \eta \in\left\{ \pm \epsilon_{i} \mid 1 \leq i \leq m\right\} \text { or } \zeta, \eta \in\left\{ \pm \delta_{j} \mid 1 \leq j \leq n\right\} \text {, } \\
& \mathscr{S}_{\zeta, \eta, p, q}:=r_{\zeta-\eta+p \delta} r_{\zeta-\eta+q \delta}, \quad \text { and } \quad \mathscr{T}_{\zeta, \eta, p, q}:=r_{\zeta+\eta+p \delta} r_{\zeta-\eta+q \delta} \text {. }
\end{aligned}
$$

Then for $w \in\left\{\mathscr{I}_{2 \zeta, p}, \mathscr{S}_{\zeta, \eta, p, q}, \mathscr{T}_{\zeta, \eta, p, q}, \mathscr{J}_{\zeta, \eta, p}\right\}, w(R) \cup w^{-1}(R) \subseteq R$; in particular, if $\Pi$ is a base of $R$, then $w(\Pi)$ is also a base of $R$.

Proof. It easily follows using a direct verification together with Lemma 2.4.

\subsection{Type $A(2 m, 2 n)^{(4)}$. Set}

$$
\begin{array}{rlr}
\dot{R} & := \pm\left\{\epsilon_{i}, \delta_{j}, 2 \epsilon_{i}, 2 \delta_{j}, \epsilon_{i} \pm \epsilon_{r}, \delta_{j} \pm \delta_{s}, \delta_{j} \pm \epsilon_{i} \mid 1 \leq i \neq r \leq m, 1 \leq j \neq s \leq n\right\} \\
\dot{R}_{s h}: & :=\left\{ \pm \epsilon_{i}, \pm \delta_{p} \mid 1 \leq i \leq m, 1 \leq p \leq n\right\} & \text { (short roots), } \\
\dot{R}_{l g}: & :=\left\{ \pm \epsilon_{i} \pm \epsilon_{j}, \pm \delta_{p} \pm \delta_{q} \mid 1 \leq i \neq j \leq m, 1 \leq p \neq q \leq n\right\} & \text { (long roots), } \\
\dot{R}_{e x}:=\left\{ \pm 2 \epsilon_{i}, \pm 2 \delta_{p} \mid 1 \leq i \leq m, 1 \leq p \leq n\right\} & \text { (extra long roots), } \\
\dot{R}_{n s}:=\left\{ \pm \epsilon_{i} \pm \delta_{p} \mid 1 \leq i \leq m, 1 \leq p \leq n\right\} &
\end{array}
$$

and

$$
\left.R:=\mathbb{Z} \delta \cup\left(\dot{R}_{s h}+\mathbb{Z} \delta\right) \cup\left(\dot{R}_{l g} \cup \dot{R}_{n s}\right)+2 \mathbb{Z} \delta\right) \cup\left(\left\{ \pm 2 \epsilon_{i}\right\}_{i=1}^{m}+4 \mathbb{Z} \delta+2 \delta\right) \cup\left(\left\{ \pm 2 \delta_{p}\right\}_{p=1}^{n}+4 \mathbb{Z} \delta\right) .
$$

For

$$
S:=\mathbb{Z} \delta \cup\left(\dot{R}_{s h}+\mathbb{Z} \delta\right) \cup\left(\left(\dot{R}_{l g} \cup \dot{R}_{n s}\right)+2 \mathbb{Z} \delta\right),
$$

$\left(\operatorname{span}_{\mathbb{R}} S,(\cdot, \cdot)_{*}, S\right)$ is the root system of $D_{m+n+1}^{(2)}$. Using Lemma 2.2, each base of $R$ is a base of $S$ and conversely each base of $S$ is a base of $R$. So for $\theta_{i}(1 \leq i \leq \ell)$ as in Theorem 1.1 (see Table 2 for further assumptions), we have

$$
S=\mathbb{Z} \delta \cup\left(\left\{ \pm \theta_{i} \mid 1 \leq i \leq \ell\right\}+\mathbb{Z} \delta\right) \cup\left(\left\{ \pm \theta_{i} \pm \theta_{j} \mid 1 \leq i \neq j \leq \ell\right\}+2 \mathbb{Z} \delta\right)
$$

and

$$
\Pi=\left\{\theta_{i}-\theta_{i+1}, \theta_{\ell}, \delta-\theta_{1}, \mid 1 \leq i \leq \ell-1\right\}
$$

is a base of $S$ (and so a base of $R$ ). Moreover, the quasi-Weyl group of $R$ is the Weyl group of $S$ and so from affine Lie theory, up to \pm 1 -multiple, all bases of $R$ are conjugate under quasi-Weyl group; in particular, all bases of $R$ are of the form $\Pi$ or $-\Pi$. Next suppose $\Delta$ is the standard base of $R$ (which is also a base of $S$ ), then again using affine Lie theory, for each base $\Delta^{\prime}$ of $S$ (or equivalently of $R$ ), we have $S^{+}(\Delta) \cap S^{-}\left(\Delta^{\prime}\right)$ or $S^{+}(\Delta) \cap S^{+}\left(\Delta^{\prime}\right)$ is finite and consequently either $R^{+}(\Delta) \cap R^{-}\left(\Delta^{\prime}\right)$ or $R^{+}(\Delta) \cap R^{+}\left(\Delta^{\prime}\right)$ is finite. Therefore, we get using Lemma 2.3 that $\Delta$ is an S-base. This in particular completes the proof of Theorem 1.1 for $R=A(2 m, 2 n)^{(4)}$.

2.2. Type $\boldsymbol{D}(\boldsymbol{m}+\mathbf{1}, \boldsymbol{n})^{(\mathbf{2})}$. One can prove Theorem 1.1 using the same argument as in the previous case. 


\subsection{Type $A(\mathbf{2} m, \mathbf{2 n}-\mathbf{1})^{(2)}$. Set}

$$
\begin{array}{rlr}
\dot{R} & :=\left\{0, \pm \epsilon_{i}, \pm \epsilon_{i} \pm \epsilon_{j}, \pm 2 \epsilon_{i}, \pm \delta_{p}, \pm \delta_{p} \pm \delta_{q}, \pm 2 \delta_{p}, \pm \epsilon_{i} \pm \delta_{p} \mid 1 \leq i \neq j \leq m, 1 \leq p \neq q \leq n\right\} \\
\dot{R}_{s h}:=\left\{ \pm \epsilon_{i}, \pm \delta_{p} \mid 1 \leq i \leq m, 1 \leq p \leq n\right\} & & \text { (short roots), } \\
\dot{R}_{l g}: & =\left\{ \pm \epsilon_{i} \pm \epsilon_{j}, \pm \delta_{p} \pm \delta_{q} \mid 1 \leq i \neq j \leq m, 1 \leq p \neq q \leq n\right\} & \text { (long roots) } \\
\dot{R}_{e x}:=\left\{ \pm 2 \epsilon_{i}, \pm 2 \delta_{p} \mid 1 \leq i \leq m, 1 \leq p \leq n\right\} & & \text { (extra-long roots), } \\
\dot{R}_{n s}:=\left\{ \pm \epsilon_{i} \pm \delta_{p}, 1 \leq i \leq m, 1 \leq p \leq n\right\} . &
\end{array}
$$

and

$$
R:=\mathbb{Z} \delta \cup\left(\left(\dot{R} \backslash \dot{R}_{e x}\right)+\mathbb{Z} \delta\right) \cup\left(\left\{ \pm 2 \epsilon_{i}\right\}_{i=1}^{m}+2 \mathbb{Z} \delta+\delta\right) \cup\left(\left\{ \pm 2 \delta_{p}\right\}_{p=1}^{n}+2 \mathbb{Z} \delta\right) .
$$

We recall that

$$
\ell=m+n
$$

and set

$$
\begin{aligned}
& \dot{S}:=\dot{R} \backslash \dot{R}_{e x}=\left\{0, \pm \zeta, \pm \zeta \pm \xi \mid \zeta \neq \xi \in\left\{\epsilon_{i}, \delta_{p} \mid 1 \leq i \leq m, 1 \leq p \leq n\right\}\right\} \\
& S:=\dot{S}+\mathbb{Z} \delta \text { and } \\
& T:=S \cup\left(\left\{ \pm 2 \zeta \mid \zeta \in\left\{\epsilon_{i}, \delta_{p} \mid 1 \leq i \leq m, 1 \leq p \leq n\right\}\right\}+2 \mathbb{Z} \delta+\delta\right) .
\end{aligned}
$$

Then $\left(V,(\cdot, \cdot)_{*}, S\right)$ is the root system of $B_{m+n}^{(1)}$ and $\left(V,(\cdot, \cdot)_{*}, T\right)$ is the root system of $A_{2(m+n)}^{(2)}$. We have

(2.3) $T \backslash R=\left\{ \pm 2 \delta_{p} \mid 1 \leq p \leq n\right\}+2 \mathbb{Z} \delta+\delta \quad$ and $\quad R \backslash T=\left\{ \pm 2 \delta_{p} \mid 1 \leq p \leq n\right\}+2 \mathbb{Z} \delta$.

In what follows, we call elements of $\left(\left\{ \pm 2 \epsilon_{i}\right\}_{i=1}^{n}+2 \mathbb{Z} \delta\right) \cup\left(\left\{ \pm 2 \delta_{p}\right\}_{p=1}^{m}+2 \mathbb{Z} \delta+\delta\right)$, extra-long roots of $R$ and denote by $W$ the quasi-Weyl group of $R$.

Proposition 2.6. If $\Pi \subseteq R$ is a base of $T$, then $\Pi$ is a base of $R$ and conversely, each base of $R$ containing an extra long root is a base of $T$.

Proof. Suppose that $\Pi \subseteq R$ is a base of $T$. Since for each $1 \leq p \leq n, 2 \delta_{p}+2 \mathbb{Z} \delta=$ $2\left(\delta_{p}+\mathbb{Z} \delta\right) \subseteq 2 T, \Pi$ is a base of $R$.

Conversely, suppose that $\Pi$ is a base of $R$ containing an extra long root $\alpha$, then by Lemma 2.2, $\alpha=2 r \epsilon_{i}+2 k \delta+\delta$ for some $r \in\{ \pm 1\}, 1 \leq i \leq m$ and $k \in \mathbb{Z}$. Set $\dot{\epsilon}_{i}:=-r \epsilon_{i}-k \delta \in R$, so

$$
\alpha=-2 \dot{\epsilon}_{i}+\delta \in \Pi \text {. }
$$

To the contrary assume $\Pi$ is not a base of $T$. Using (2.3), there is an index $p_{0}$, $s \in\{ \pm 1\}$ and $k^{\prime} \in \mathbb{Z}$ such that $2 s \delta_{p_{0}}+\left(2 k^{\prime} \delta+\delta\right) \notin \operatorname{span}_{\mathbb{Z} \geq 0} \Pi \cup \operatorname{span}_{\mathbb{Z} \leq 0} \Pi$. Set $\dot{\delta}_{p_{0}}:=s \delta_{p_{0}}+k^{\prime} \delta \in R$. So

$$
2 \dot{\delta}_{p_{0}}+\delta \notin \operatorname{span}_{\mathbb{Z} \geq 0} \Pi \cup \operatorname{span}_{\mathbb{Z} \leq 0} \Pi \text {. }
$$

Since $\Pi$ is a basis of $V$, by Lemma 2.2, there is an element of $\Pi$ of the form $t \dot{\delta}_{p_{0}}+\rho$ for some $t \in\{ \pm 1\}$ and $\rho \in \mathbb{Z} \delta \cup\left(\left\{ \pm \delta_{q} \mid 1 \leq q \neq p_{0} \leq n\right\}+\mathbb{Z} \delta\right) \cup\left(\left\{ \pm \epsilon_{j} \mid 1 \leq j \leq n\right\}+\mathbb{Z} \delta\right)$. Assume $\Pi=\left\{\alpha_{1}, \ldots, \alpha_{\ell+1}\right\}$ with

$$
\alpha_{1}=-2 \dot{\epsilon}_{i}+\delta \quad \text { and } \quad \alpha_{2}=t \dot{\delta}_{p_{0}}+\rho .
$$

We know that $\dot{\delta}_{p_{0}}+\dot{\epsilon}_{i}, t \dot{\delta}_{p_{0}}-\rho+t \delta \in R$. Therefore, there are integers $r_{i}, s_{i}(1 \leq$ $i \leq \ell+1)$ such that

$$
\dot{\delta}_{p_{0}}+\dot{\epsilon}_{i}=\sum_{i=1}^{\ell+1} r_{i} \alpha_{i} \quad \text { and } \quad t \dot{\delta}_{p_{0}}-\rho+t \delta=\sum_{i=1}^{\ell+1} s_{i} \alpha_{i}
$$


and $r_{i}$ 's (resp. $s_{i}$ 's) are all non-negative or all non-positive. We have

$$
2 \dot{\delta}_{p_{0}}+\delta=\left(-2 \dot{\epsilon}_{i}+\delta\right)+2\left(\dot{\delta}_{p_{0}}+\dot{\epsilon}_{i}\right)=\alpha_{1}+\sum_{i=1}^{\ell+1} 2 r_{i} \alpha_{i}=\left(1+2 r_{1}\right) \alpha_{1}+\sum_{i=2}^{\ell+1} 2 r_{i} \alpha_{i}
$$

and

$$
2 t \dot{\delta}_{p_{0}}+t \delta=\left(t \dot{\delta}_{p_{0}}+\rho\right)+\left(t \dot{\delta}_{p_{0}}-\rho+t \delta\right)=\alpha_{2}+\sum_{i=1}^{\ell+1} s_{i} \alpha_{i}=\left(1+s_{2}\right) \alpha_{2}+\sum_{2 \neq i=1}^{\ell+1} s_{i} \alpha_{i}
$$

If $t \dot{\delta}_{p_{0}}-\rho+t \delta$ is a positive root that is for all $i, s_{i} \geq 0$, then $2 t \dot{\delta}_{p_{0}}+t \delta \in \operatorname{span}_{\mathbb{Z} \geq 0} \Pi$ contradicting (2.4). So $t \dot{\delta}_{p_{0}}-\rho+t \delta$ is a negative root; in other words $s_{i} \leq 0$ for all $i$. Using (2.4), we get that $0<1+s_{2} \leq 1$. This implies that $s_{2}=0$. Therefore,

$$
\left(1+2 r_{1}\right) \alpha_{1}+\sum_{i=2}^{\ell+1} 2 r_{i} \alpha_{i}=2 \dot{\delta}_{p_{0}}+\delta=t \alpha_{2}+\sum_{2 \neq i=1}^{\ell+1} t s_{i} \alpha_{i}
$$

which implies that $2 r_{2}=t \in\{ \pm 1\}$, a contradiction.

Lemma 2.7. Assume $\theta_{i}$ 's and $\Pi$ are as in Theorem 1.1. Then $\Pi$ is a base of $R$ and

$$
\begin{aligned}
R^{+}(\Pi) & =\left\{ \pm 2 \theta_{i} \mid \operatorname{supp}\left(\theta_{i}\right) \subseteq\left\{\epsilon_{j} \mid 1 \leq j \leq m\right\}\right\}+2 \mathbb{Z}^{\geq 0} \delta+\delta \\
& \cup\left\{ \pm 2 \theta_{i} \mid \operatorname{supp}\left(\theta_{i}\right) \subseteq\left\{\delta_{p} \mid 1 \leq p \leq n\right\}+2 \mathbb{Z}^{>0} \delta\right. \\
& \cup\left\{0, \pm \theta_{i}, \pm \theta_{i} \pm \theta_{j} \mid 1 \leq i<j \leq \ell\right\}+\mathbb{Z}^{>0} \delta \\
& \cup\left\{\theta_{i}, \theta_{i} \pm \theta_{j} \mid 1 \leq i<j \leq \ell\right\} \cup\left\{2 \theta_{i} \mid \operatorname{supp}\left(\theta_{i}\right) \subseteq\left\{\delta_{p} \mid 1 \leq p \leq n\right\}\right\} .
\end{aligned}
$$

Proof. We have two cases:

Case $1 . \Pi=\left\{\delta-2 \theta_{1}, \theta_{i}-\theta_{i+1}, \theta_{\ell} \mid 1 \leq i \leq \ell-1\right\}$ : In this case, the result follows easily from Proposition 2.6 and affine Lie theory as $\Pi$ is a base of

$$
T=\left\{0, \pm \theta_{i}, \pm \theta_{i} \pm \theta_{j} \mid 1 \leq i \neq j \leq \ell\right\}+\mathbb{Z} \delta \cup\left\{ \pm 2 \theta_{i} \mid 1 \leq i \leq \ell\right\}+2 \mathbb{Z} \delta+\delta,
$$

which is the root system of $A_{2 \ell}^{(2)}$.

Case 2. $\Pi=\left\{\delta-\left(\theta_{1}+\theta_{2}\right), \theta_{i}-\theta_{i+1}, \theta_{\ell} \mid 1 \leq i \leq \ell-1\right\}$ : We have

$$
\begin{aligned}
S & =\left\{0, \pm \epsilon_{i}, \pm \delta_{p}, \pm \epsilon_{i} \pm \epsilon_{j}, \pm \delta_{p} \pm \delta_{q}, \pm \epsilon_{i} \pm \delta_{p} \mid 1 \leq i \neq j \leq m, 1 \leq p \neq q \leq n\right\}+\mathbb{Z} \delta \\
& =\left\{0, \pm \theta_{i}, \pm \theta_{i} \pm \theta_{j} \mid 1 \leq i \neq j \leq \ell\right\}+\mathbb{Z} \delta
\end{aligned}
$$

which is the root system of $B_{\ell}^{(1)}$ and that $\Pi$ is a base of $S$ with

$$
S^{+}(\Pi)=\left\{0, \pm \theta_{i}, \pm \theta_{i} \pm \theta_{j} \mid 1 \leq i<j \leq \ell\right\}+\mathbb{Z}^{>0} \delta \cup\left\{\theta_{i}, \theta_{i} \pm \theta_{j} \mid 1 \leq i<j \leq \ell\right\} .
$$

So we have

$$
-2 \theta_{2}+\delta=\theta_{1}-\theta_{2}+\left(-\left(\theta_{1}+\theta_{2}\right)+\delta\right) \in \Pi+\Pi \subseteq \operatorname{span}_{\mathbb{Z} \geq 0} \Pi .
$$

Therefore, for $t \in\{ \pm 1\}, k \in \mathbb{Z}^{\geq 0}$ and $2 \leq i \leq m+n$, we have

$$
2 t \theta_{i}+2 k \delta+\delta=2\left(t \theta_{i}+\theta_{2}\right)+\left(-2 \theta_{2}+\delta\right)+2 k \delta \in \operatorname{span}_{\mathbb{Z} \geq 0} \Pi .
$$

We are done using this together with the fact that $\operatorname{supp}\left(\theta_{1}\right) \subseteq\left\{\delta_{p} \mid 1 \leq p \leq n\right\}$.

The following theorem completes the proof of Theorem 1.1 for the case under consideration: 
Theorem 2.8. $\quad$ (i) Each base of $R$ is of the form $\pm \Pi$ where $\Pi$ is as in Table 2. In particular, if

$$
\xi_{i}:=\left\{\begin{array}{ll}
\delta_{i} & 1 \leq i \leq n, \\
\epsilon_{i-n} & n+1 \leq i \leq \ell,
\end{array} \eta_{i}:= \begin{cases}\epsilon_{i} & 1 \leq i \leq m \\
\delta_{i-m} & m+1 \leq i \leq \ell\end{cases}\right.
$$

then

$$
\Pi_{1}:=\left\{\delta-2 \eta_{1}, \eta_{i}-\eta_{i+1}, \eta_{\ell}\right\} \quad \text { and } \quad \Pi_{2}:=\left\{\delta-\left(\xi_{1}+\xi_{2}\right), \xi_{i}-\xi_{i+1}, \xi_{\ell}\right\}
$$

are bases of $R$ and up to conjugacy under quasi-Weyl group, $\pm \Pi_{1}$ and $\pm \Pi_{2}$ are the only bases of $R$.

(ii) Each base of $R$ is an $S$-base.

Proof. (i) Suppose that $B$ is a base of $R$. Two cases can happen: Either $B$ contains an extra long root or it is contained in $S$.

Case 1. $B$ contains an extra long root: We first mention that $\Pi_{1}$ is a base of $T$ and that under the Weyl group action of $T$, each base of $T$ is conjugate with $\Pi_{1}$ or $-\Pi_{1}$. If $W_{1}$ is the Weyl group of $T$, then $W_{1}$ equals with the group generated by the reflections based on the elements of $\Pi_{1}$. So $W_{1} \subseteq W$.

By Proposition 2.6] B is a base of $T$; in particular, there is an element $w \in W_{1} \subseteq$ $W$ such that either $w\left(\Pi_{1}\right)=B$ or $w\left(\Pi_{1}\right)=-B$. Set

$$
\theta_{i}:=w\left(\eta_{i}\right) \in\left\{ \pm \epsilon_{j}, \pm \delta_{p} \mid 1 \leq j \leq m, 1 \leq p \leq n\right\}+\mathbb{Z} \delta \quad(1 \leq i \leq \ell) .
$$

Since $-2 \theta_{1}+\delta=w\left(-2 \eta_{1}+\delta\right) \in \pm B \subseteq R$, we get that $\theta_{1} \in\left\{ \pm \epsilon_{i} \mid 1 \leq i \leq m\right\}+\mathbb{Z} \delta$. Also we have

$$
\pm B=w\left(\Pi_{1}\right)=\left\{\theta_{1}-\theta_{2}, \ldots, \theta_{\ell-1}-\theta_{\ell}, \theta_{\ell}, \delta-2 \theta_{1}\right\} .
$$

Case 2. $B$ is a base of $R$ contained in $S$ : In this case, $B$ is a base of $S$; in particular, $B$ is either conjugate with $\Pi_{2}$ or $-\Pi_{2}$ under the Weyl group action of $S$. But Weyl group of $S$ is a subgroup of $W$, so there is $w \in W$ with $w\left(\Pi_{2}\right)= \pm B$. Set

$$
\theta_{i}:=w\left(\eta_{i}\right) \in\left\{ \pm \epsilon_{t}, \pm \delta_{p} \mid 1 \leq t \leq m, 1 \leq p \leq n\right\}+\mathbb{Z} \delta \quad(1 \leq i \leq \ell) .
$$

So

We have

$$
\pm B=w\left(\Pi_{2}\right)=\left\{\theta_{1}-\theta_{2}, \ldots, \theta_{\ell-1}-\theta_{\ell}, \theta_{\ell}, \delta-\left(\theta_{1}+\theta_{2}\right)\right\} .
$$

$$
-2 \theta_{1}+\delta=-\left(\theta_{1}-\theta_{2}\right)+\left(\delta-\left(\theta_{1}+\theta_{2}\right)\right) \in-\Pi_{2}+\Pi_{2} .
$$

This means that $-2 \theta_{1}+\delta \notin R$, i.e., $\theta_{1} \in\left\{ \pm \delta_{p} \mid 1 \leq p \leq n\right\}+\mathbb{Z} \delta$. This completes the proof.

(ii) Suppose that $\Delta$ is the standard base of $R$, then by part (i) and Lemma 2.7 for each base $\Delta^{\prime}$, either $R^{+}(\Delta) \cap R^{-}\left(\Delta^{\prime}\right)$ or $R^{+}(\Delta) \cap R^{+}\left(\Delta^{\prime}\right)$ is finite. Therefore, we get the result using Lemma 2.3

2.4. Type $\boldsymbol{A}(\mathbf{2} \boldsymbol{m}-\mathbf{1}, \mathbf{2 n}-\mathbf{1})^{(\mathbf{2})}$. Suppose that $m, n$ are two positive integers with $(m, n) \neq(1,1)$ and assume $R$ is the root system of $A(2 m-1,2 n-1)^{(2)}$; in other words,

$$
R=\left(\left\{0, \pm \epsilon_{i} \pm \epsilon_{j}, \pm \delta_{p} \pm \delta_{q}, \pm \epsilon_{i} \pm \delta_{p}\right\}_{i, p}+\mathbb{Z} \delta\right) \cup\left(\left\{ \pm 2 \epsilon_{i}\right\}_{i}+2 \mathbb{Z} \delta+\delta\right) \cup\left(\left\{ \pm 2 \delta_{p}\right\}_{p}+2 \mathbb{Z} \delta\right)
$$

where $i \neq j$ run over $\{1, \ldots, m\}$ and $p \neq q$ run over $\{1, \ldots, n\}$. Set

$$
R_{l g}:=\left(\left\{ \pm 2 \epsilon_{i}\right\}_{i=1}^{m}+2 \mathbb{Z} \delta+\delta\right) \cup\left(\left\{ \pm 2 \delta_{p}\right\}_{p=1}^{n}+2 \mathbb{Z} \delta\right) \quad \text { (long roots) }
$$

and recall $(\cdot, \cdot)_{*}$ from $(\boxplus$ in Introduction and that

$$
\ell=m+n .
$$


Proposition 2.9. Recall $\theta_{i}$ 's and $\Pi$ from Theorem 1.1.

(i) If $\Pi=\left\{\delta-\left(\theta_{1}+\theta_{2}\right), \theta_{i}-\theta_{i+1}, \theta_{\ell-1}+\theta_{\ell} \mid 1 \leq i \leq \ell-1\right\}$, then $\Pi$ is a base of $R$ and

$$
\begin{aligned}
R^{+}(\Pi) & =\left\{\theta_{i} \pm \theta_{j} \mid i<j\right\} \cup\left\{2 \theta_{i} \mid \operatorname{supp}\left(\theta_{i}\right) \subseteq\left\{\delta_{p} \mid 1 \leq j \leq n\right\}\right\} \\
& \cup\left\{0, \pm \theta_{i} \pm \theta_{j} \mid i \neq j\right\}+\mathbb{Z}^{>0} \delta \\
& \cup\left\{ \pm 2 \theta_{i} \mid \operatorname{supp}\left(\theta_{i}\right) \subseteq\left\{\epsilon_{j} \mid 1 \leq j \leq m\right\}\right\}+2 \mathbb{Z}^{\geq 0} \delta+\delta \\
& \cup\left\{ \pm 2 \theta_{i} \mid \operatorname{supp}\left(\theta_{i}\right) \subseteq\left\{\delta_{p} \mid 1 \leq j \leq n\right\}\right\}+2 \mathbb{Z}^{>0} \delta .
\end{aligned}
$$

(ii) If $\Pi=\left\{-2 \theta_{1}, \theta_{i}-\theta_{i+1}, \theta_{\ell-1}+\theta_{\ell}+\delta \mid 1 \leq i \leq \ell-1\right\}$, then $\Pi$ is a base of $R$ and

$$
\begin{aligned}
R^{+}(\Pi) & =\left\{\theta_{i}-\theta_{j},-\theta_{i}-\theta_{j} \mid i<j\right\} \cup\left\{-2 \theta_{i} \mid \operatorname{supp}\left(\theta_{i}\right) \subseteq\left\{\delta_{p} \mid 1 \leq j \leq n\right\}\right\} \\
& \cup\left\{0, \pm \theta_{i} \pm \theta_{j} \mid i \neq j\right\}+\mathbb{Z}^{>0} \delta \\
& \cup\left\{ \pm 2 \theta_{i} \mid \operatorname{supp}\left(\theta_{i}\right) \subseteq\left\{\epsilon_{j} \mid 1 \leq j \leq m\right\}\right\}+2 \mathbb{Z}^{\geq 0} \delta+\delta \\
& \cup\left\{ \pm 2 \theta_{i} \mid \operatorname{supp}\left(\theta_{i}\right) \subseteq\left\{\delta_{p} \mid 1 \leq j \leq n\right\}\right\}+2 \mathbb{Z}^{>0} \delta .
\end{aligned}
$$

(iii) If $\Pi=\left\{-2 \theta_{1}+\delta, \theta_{i}-\theta_{i+1}, \theta_{\ell-1}+\theta_{\ell} \mid 1 \leq i \leq \ell-1\right\}$, then $\Pi$ is a base of $R$ and

$$
\begin{aligned}
R^{+}(\Pi) & =\left\{\theta_{i} \pm \theta_{j} \mid i<j\right\} \cup\left\{2 \theta_{i} \mid \operatorname{supp}\left(\theta_{i}\right) \subseteq\left\{\delta_{p} \mid 1 \leq j \leq n\right\}\right\} \\
& \cup\left\{0, \pm \theta_{i} \pm \theta_{j} \mid i \neq j\right\}+\mathbb{Z}^{>0} \delta \\
& \cup\left\{ \pm 2 \theta_{i} \mid \operatorname{supp}\left(\theta_{i}\right) \subseteq\left\{\epsilon_{j} \mid 1 \leq j \leq m\right\}\right\}+2 \mathbb{Z}^{\geq 0} \delta+\delta \\
& \cup\left\{ \pm 2 \theta_{i} \mid \operatorname{supp}\left(\theta_{i}\right) \subseteq\left\{\delta_{p} \mid 1 \leq j \leq n\right\}\right\}+2 \mathbb{Z}^{>0} \delta .
\end{aligned}
$$

(iv) If $\Pi=\left\{-2 \theta_{1}, \theta_{i}-\theta_{i+1}, 2 \theta_{\ell}+\delta \mid 1 \leq i \leq \ell-1\right\}$, then $\Pi$ is a base of $R$ and

$$
\begin{aligned}
R^{+}(\Pi) & =\left\{\theta_{i}-\theta_{j},-\theta_{i}-\theta_{j} \mid i<j\right\} \cup\left\{-2 \theta_{i} \mid \operatorname{supp}\left(\theta_{i}\right) \subseteq\left\{\delta_{p} \mid 1 \leq j \leq n\right\}\right\} \\
& \cup\left\{0, \pm \theta_{i} \pm \theta_{j} \mid i \neq j\right\}+\mathbb{Z}^{>0} \delta \\
& \cup\left\{ \pm 2 \theta_{i} \mid \operatorname{supp}\left(\theta_{i}\right) \subseteq\left\{\epsilon_{j} \mid 1 \leq j \leq m\right\}\right\}+2 \mathbb{Z}^{\geq 0} \delta+\delta \\
& \cup\left\{ \pm 2 \theta_{i} \mid \operatorname{supp}\left(\theta_{i}\right) \subseteq\left\{\delta_{p} \mid 1 \leq j \leq n\right\}\right\}+2 \mathbb{Z}^{>0} \delta .
\end{aligned}
$$

Proof. (i) Setting

$$
\begin{aligned}
S: & = \pm\left\{0, \theta_{i} \pm \theta_{j} \mid 1 \leq i \neq j \leq \ell\right\}+\mathbb{Z} \delta \\
& = \pm\left\{0, \epsilon_{i} \pm \epsilon_{j}, \delta_{p} \pm \delta_{q}, \epsilon_{i} \pm \delta_{p} \mid 1 \leq i \neq j \leq m, 1 \leq p \neq q \leq n\right\}+\mathbb{Z} \delta,
\end{aligned}
$$

we get that $\left(\operatorname{span}_{\mathbb{R}} S,(\cdot, \cdot)_{*}, S\right)$ is the root system of $D_{\ell}^{(1)}$ and $\Pi$ is a base of $S$. One knows that the set $S^{+}$of positive roots of $S$ with respect to $\Pi$ is

$$
S^{+}=\left\{\theta_{i} \pm \theta_{j} \mid 1 \leq i<j \leq \ell\right\} \cup\left( \pm\left\{0, \theta_{i} \pm \theta_{j} \mid 1 \leq i \neq j \leq \ell\right\}+\mathbb{Z}^{>0} \delta\right) .
$$

To complete the proof, it is enough to show that for all $1 \leq i \leq \ell$,

- $\pm 2 \theta_{i}+2 \mathbb{Z} \geq 0 \delta+\delta \in \operatorname{span}_{\mathbb{Z} \geq 0} \Pi$ if $\operatorname{supp}\left(\theta_{i}\right) \subseteq\left\{\epsilon_{j} \mid 1 \leq j \leq m\right\}$ and

- $2 \theta_{i}, \pm 2 \theta_{i}+2 \mathbb{Z}^{>0} \delta \in \operatorname{span}_{\mathbb{Z} \geq 0} \Pi$ if $\operatorname{supp}\left(\theta_{i}\right) \subseteq\left\{\delta_{p} \mid 1 \leq p \leq n\right\}$.

We first assume $\operatorname{supp}\left(\theta_{i}\right) \subseteq\left\{\epsilon_{j} \mid 1 \leq j \leq m\right\}$. Then $i \neq 1$ and so for $k \geq 0$, using (2.9), we have

$$
\pm 2 \theta_{i}+(2 k+1) \delta=\left(-\theta_{1} \pm \theta_{i}+(2 k+1) \delta\right)+\left(\theta_{1} \pm \theta_{i}\right) \in \operatorname{span}_{\mathbb{Z} \geq 0} \Pi .
$$


Also if $\operatorname{supp}\left(\theta_{i}\right) \subseteq\left\{\delta_{p} \mid 1 \leq p \leq n\right\}$, we have $i \neq \ell$ and so by (2.9), we have

$$
\pm 2 \theta_{i}+2 k \delta=\left( \pm \theta_{i}+\theta_{\ell}+k \delta\right)+\left( \pm \theta_{i}-\theta_{\ell}+k \delta\right) \in \operatorname{span}_{\mathbb{Z} \geq 0} \Pi \quad(k>0)
$$

and

$$
2 \theta_{i}=\left(\theta_{i}-\theta_{\ell}\right)+\left(\theta_{i}+\theta_{\ell}\right) \in \operatorname{span}_{\mathbb{Z} \geq 0} \Pi
$$

and so we are done.

(ii)-(iv) Suppose $\Pi$ is as in the statements. To simplify our argument, we also denote the $\Pi$ introduced in (ii), (iii) and (iv) respectively by $B^{2}, B^{3}$ and $B^{4}$. Let

\begin{tabular}{|c|c|c|}
\hline$\theta_{i}-\theta_{j} \quad(i<j)$ & $\left(\theta_{i}-\theta_{i+1}\right)+\cdots+\left(\theta_{j-1}-\theta_{j}\right)$ & $\in \operatorname{span}_{\mathbb{Z} \geq 0} \Pi$ \\
\hline$\theta_{i}+\theta_{j} \quad(i<j)$ & 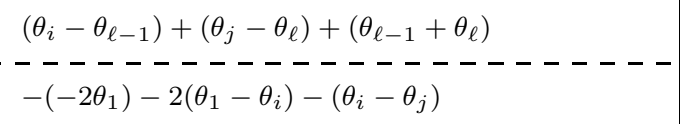 & $\begin{array}{c}\in \operatorname{span}_{\mathbb{Z} \geq 0} B^{3} \\
\in------------- \\
\in \operatorname{span}_{\mathbb{Z} \leq 0} B^{2} \cup \operatorname{span}_{\mathbb{Z} \leq 0} B^{4}\end{array}$ \\
\hline$\theta_{i}+\theta_{j}+\delta(i<j)$ & $\begin{array}{l}\left(\theta_{i}-\theta_{\ell-1}\right)+\left(\theta_{j}-\theta_{\ell}\right)+\left(\theta_{\ell-1}+\theta_{\ell}+\delta\right) \\
-------------------- \\
\left(\theta_{i}+\theta_{j}\right)+\left(\theta_{1}+\theta_{2}\right)+\left(\theta_{1}-\theta_{2}\right)+\left(-2 \theta_{1}+\delta\right) \\
--------------------- \\
\left(\theta_{i}-\theta_{\ell}\right)+\left(\theta_{j}-\theta_{\ell}\right)+\left(2 \theta_{\ell}+\delta\right)\end{array}$ & 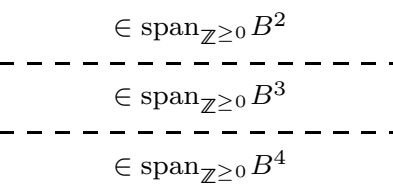 \\
\hline $2 \theta_{i}+\delta$ & $\begin{array}{l}\left(\theta_{i}-\theta_{i+1}\right)+\left(\theta_{i}+\theta_{i+1}+\delta\right) \quad(\text { if } i \neq \ell) \\
-------------------- \\
2\left(\theta_{i}+\theta_{i+1}\right)+2\left(\theta_{1}-\theta_{i+1}\right)+\left(-2 \theta_{1}+\delta\right) \quad(\text { if } i \neq \ell) \\
\left(-2 \theta_{1}+\delta\right)+2\left(\theta_{1}+\theta_{\ell}\right) \quad(\text { if } i=\ell) \\
------------------- \\
2\left(\theta_{i}-\theta_{\ell}\right)+\left(2 \theta_{\ell}+\delta\right)\end{array}$ & 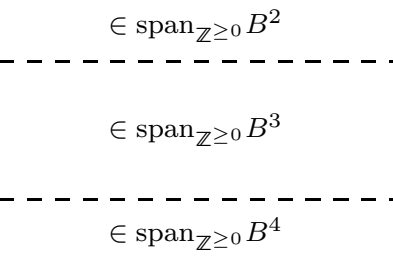 \\
\hline$-2 \theta_{i}$ & $\begin{array}{l}-\left(\theta_{i}-\theta_{i+1}\right)-\left(\theta_{i}+\theta_{i+1}\right) \quad(\text { if } i \neq \ell) \\
--------------------- \\
\left(-2 \theta_{1}\right)+2\left(\theta_{1}-\theta_{2}\right)+\cdots+2\left(\theta_{i-1}-\theta_{i}\right)\end{array}$ & $\begin{array}{c}\in \operatorname{span}_{\mathbb{Z} \leq 0} B^{3} \\
------------\cdot \\
\in \operatorname{span}_{\mathbb{Z} \geq 0} B^{2} \cup \operatorname{span}_{\mathbb{Z} \geq 0} B^{4}\end{array}$ \\
\hline$\delta$ & $\begin{array}{l}\left(\theta_{1}+\theta_{2}\right)+\left(\theta_{1}-\theta_{2}\right)+\left(-2 \theta_{1}+\delta\right) \\
--------------------- \\
\left(2 \theta_{1}+\delta\right)+\left(-2 \theta_{1}\right)\end{array}$ & $\begin{array}{c}\in \operatorname{span}_{\mathbb{Z} \geq 0} B^{3} \\
-\overline{-}-----\overline{-}----- \\
\in \operatorname{span}_{\mathbb{Z} \geq 0} B^{2} \cup \operatorname{span}_{\mathbb{Z} \geq 0} B^{4}\end{array}$ \\
\hline$\theta_{i}+\theta_{j}+k \delta$ & $\begin{array}{l}\left(\theta_{i}+\theta_{j}\right)+k \delta \\
---\overline{-}----------------- \\
\left(\theta_{i}+\theta_{j}+\delta\right)+(k-1) \delta\end{array}$ & $\begin{array}{c}\in \operatorname{span}_{\mathbb{Z} \geq 0} B^{3} \\
\in------------ \\
\in \operatorname{span}_{\mathbb{Z} \geq 0} B^{2} \cup \operatorname{span}_{\mathbb{Z} \geq 0} B^{4}\end{array}$ \\
\hline$-2 \theta_{i}+k \delta$ & $\begin{array}{l}\left(-2 \theta_{1}+\delta\right)+2\left(\theta_{1}-\theta_{i}\right)+(k-1) \delta \\
--------------------- \\
\left(-2 \theta_{i}\right)+k \delta\end{array}$ & $\begin{array}{c}\in \operatorname{span}_{\mathbb{Z} \geq 0} B^{3} \\
\qquad------------ \\
\in \operatorname{span}_{\mathbb{Z} \geq 0} B^{2} \cup \operatorname{span}_{\mathbb{Z} \geq 0} B^{4}\end{array}$ \\
\hline$\theta_{i}-\theta_{j}+k \delta$ & $\begin{array}{l}\left(\theta_{i}+\theta_{j}\right)+\left(-2 \theta_{j}+\delta\right)+(k-1) \delta \\
---\overline{-}---------------- \\
\left(\theta_{i}+\theta_{j}+\delta\right)+\left(-2 \theta_{j}\right)+(k-1) \delta\end{array}$ & $\begin{array}{c}\in \operatorname{span}_{\mathbb{Z} \geq 0} B^{3} \\
\in \operatorname{span}_{\mathbb{Z} \geq 0} B^{2} \cup \operatorname{span}_{\mathbb{Z} \geq 0} B^{4}\end{array}$ \\
\hline $2 \theta_{i}+k \delta \quad(k \geq 2)$ & $\begin{array}{l}\left(2 \theta_{i}+\delta\right)+(k-1) \delta \\
-------------------- \\
\left(\theta_{i}-\theta_{1}+(k-1) \delta\right)+\left(\theta_{i}+\theta_{1}+\delta\right)\end{array}$ & $\begin{array}{l}\in \operatorname{span}_{\mathbb{Z} \geq 0} B^{3} \cup \operatorname{span}_{\mathbb{Z} \geq 0} B^{4} \\
\quad \in \operatorname{span}_{\mathbb{Z} \geq 0} B^{2}\end{array}$ \\
\hline$\theta_{i}+\theta_{j}-k \delta \quad(i<j)$ & $-\left(\theta_{i}-\theta_{j}\right)-\left(-2 \theta_{i}+k \delta\right)$ & $\in \operatorname{span}_{\mathbb{Z} \leq 0} \Pi$ \\
\hline
\end{tabular}
$k \in \mathbb{Z}^{>0}$ and $1 \leq i \neq j \leq \ell$, then we have the following table:

This completes the proof. 
We shall show that the bases introduced in Proposition 2.9 are the only bases of $R$. Let us start with stating two facts which are easily verified and we use them frequently in the sequel:

Fact 1. If $\alpha, \beta \in R$ with $(\alpha, \beta)_{*}=0$ and $\operatorname{supp}(\alpha) \cap \operatorname{supp}(\beta) \neq \emptyset$, then $\operatorname{supp}(\alpha)=$ $\operatorname{supp}(\beta)$ and $|\operatorname{supp}(\alpha)|=|\operatorname{supp}(\beta)|=2$, in which $|\cdot|$ indicates the cardinal number; moreover, if $\operatorname{supp}(\alpha)=\left\{\zeta_{1}, \zeta_{2}\right\}$, then

$$
\operatorname{sgn}\left(\zeta_{1} ; \alpha\right) \operatorname{sgn}\left(\zeta_{2} ; \alpha\right)=-\operatorname{sgn}\left(\zeta_{1} ; \beta\right) \operatorname{sgn}\left(\zeta_{2} ; \beta\right) .
$$

Fact 2. If $\alpha, \beta \in R$ such that $\operatorname{supp}(\alpha) \neq \operatorname{supp}(\beta)$ and $\operatorname{supp}(\alpha) \cap \operatorname{supp}(\beta)=\{\epsilon\}$, then $\alpha-\beta \in R$ if and only if $\operatorname{sgn}(\epsilon ; \alpha)=\operatorname{sgn}(\epsilon ; \beta)$.

Lemma 2.10. Suppose $\Pi$ is a base of $R$. Then $\Pi$ intersects $\left\{ \pm 2 \epsilon_{i}\right\}_{i=1}^{m}+2 \mathbb{Z} \delta+\delta$ (resp. $\left\{ \pm 2 \delta_{p}\right\}_{p=1}^{n}+2 \mathbb{Z} \delta$ ) in at most one element.

Proof. Suppose to the contrary that $\alpha:=2 s \delta_{i}+2 k \delta$ and $\beta:=2 s^{\prime} \delta_{j}+2 k^{\prime} \delta$, for some $1 \leq i, j \leq n, s, s^{\prime} \in\{ \pm 1\}$ and $k, k^{\prime} \in \mathbb{Z}$, are two distinct elements of $\Pi$. We have

$$
\begin{aligned}
\left(s^{\prime} / 2\right) \alpha-(s / 2) \beta & =\left(s^{\prime} / 2\right)\left(2 s \delta_{i}+2 k \delta\right)-(s / 2)\left(2 s^{\prime} \delta_{j}+2 k^{\prime} \delta\right) \\
& =s s^{\prime}\left(\delta_{i}-\delta_{j}\right)+\left(s^{\prime} k-s k^{\prime}\right) \delta \in\{0\} \cup\left(R \backslash\{0\} \cap \frac{1}{2}\left(\operatorname{span}_{\mathbb{Z}} \Pi\right)\right)
\end{aligned}
$$

which is a contradiction. Also if $\alpha:=2 s \epsilon_{i}+2 k \delta+s \delta$ and $\beta:=2 s^{\prime} \epsilon_{j}+2 k^{\prime} \delta+s^{\prime} \delta$, for some $1 \leq i, j \leq m, s, s^{\prime} \in\{ \pm 1\}$ and $k, k^{\prime} \in \mathbb{Z}$, are two distinct elements of $\Pi$, then as above

$$
\left(s^{\prime} / 2\right) \alpha-(s / 2) \beta \in\{0\} \cup\left(R \backslash\{0\} \cap \frac{1}{2}\left(\operatorname{span}_{\mathbb{Z}} \Pi\right)\right)
$$

which is a contradiction. This completes the proof.

Lemma 2.11. Suppose that $\alpha, \beta_{1}, \beta_{2}, \beta_{3} \in R$ such that for $1 \leq i \leq 3,\left(\alpha, \beta_{i}\right)_{*} \neq 0$ and with respect to $(\cdot, \cdot)_{*}, \beta_{1}, \beta_{2}$ and $\beta_{3}$ are mutually orthogonal. Then there are $1 \leq i, j \leq 3$ with $i \neq j$ such that $\operatorname{supp}\left(\beta_{i}\right)=\operatorname{supp}\left(\beta_{j}\right)$ and $\left|\operatorname{supp}\left(\beta_{i}\right)\right|=\left|\operatorname{supp}\left(\beta_{j}\right)\right|=$ 2 .

Proof. Since for each $1 \leq i \leq 3,\left(\alpha, \beta_{i}\right)_{*} \neq 0$, we have $\operatorname{supp}(\alpha) \cap \operatorname{supp}\left(\beta_{i}\right) \neq \emptyset$. But $|\operatorname{supp}(\alpha)| \leq 2$, so there are $i, j$ with $i \neq j$ such that $\operatorname{supp}(\alpha) \cap \operatorname{supp}\left(\beta_{i}\right) \cap \operatorname{supp}\left(\beta_{j}\right) \neq$ $\emptyset$. This together with Fact 1 completes the proof.

Corollary 2.12. There is no subset $S$ of $R$ such that $\left(\operatorname{span}_{\mathbb{R}} S,(\cdot, \cdot)_{*}, S\right)$ is an irreducible finite root system of type $E_{6,7,8}$.

Proof. To the contrary, assume there is a subset $S$ of $R$ such that $\left(\operatorname{span}_{\mathbb{R}} S,(\cdot, \cdot)_{*}, S\right)$ is a finite root system of type $E_{6,7,8}$. The Dynkin diagram of $S$ has a sub-diagram as

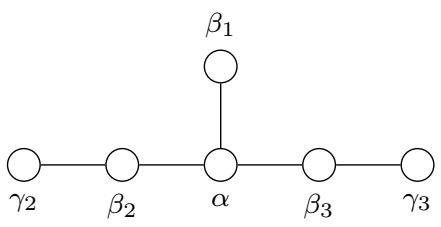


By Lemma 2.11, there are $1 \leq i \neq j \leq 3$ with $\operatorname{supp}\left(\beta_{i}\right)=\operatorname{supp}\left(\beta_{j}\right)$ and $\left|\operatorname{supp}\left(\beta_{i}\right)\right|=$ $\left|\operatorname{supp}\left(\beta_{j}\right)\right|=2$. Without loss of generality, we assume $i=2$ and assume $\operatorname{supp}\left(\beta_{2}\right)=$ $\{\epsilon, \eta\}$. Since $\left(\beta_{2}, \gamma_{2}\right)_{*} \neq 0, \operatorname{supp}\left(\gamma_{2}\right) \cap \operatorname{supp}\left(\beta_{2}\right) \neq \emptyset$. If $\operatorname{supp}\left(\gamma_{2}\right)=\operatorname{supp}\left(\beta_{2}\right)$, then $\left(\beta_{2}, \gamma_{2}\right)_{*} \neq 0$ implies that there is $r \in\{ \pm 1\}$ such that

$$
\operatorname{sgn}\left(\epsilon ; \beta_{2}\right)=r \operatorname{sgn}\left(\epsilon ; \gamma_{2}\right) \text { and } \operatorname{sgn}\left(\eta ; \beta_{2}\right)=r \operatorname{sgn}\left(\eta ; \gamma_{2}\right) .
$$

This implies that $\beta_{2}-r \gamma_{2} \in \mathbb{Z} \delta$. But $(\cdot, \cdot)_{*}$ is nondegenerate on $\operatorname{span}_{\mathbb{R}} S$, so $\beta_{2}=r \gamma_{2}$ which is a contradiction. Therefore, $\left|\operatorname{supp}\left(\gamma_{2}\right) \cap \operatorname{supp}\left(\beta_{2}\right)\right|=1$ which together with the fact that $\operatorname{supp}\left(\beta_{2}\right)=\operatorname{supp}\left(\beta_{j}\right)$ implies that $\left(\gamma_{2}, \beta_{j}\right)_{*} \neq 0$, a contradiction.

Lemma 2.13. Suppose that $B$ is a subset of a base of $R$. Assume $\alpha, \beta \in B$ such that $(\alpha, \beta)_{*}=0$ and $\operatorname{supp}(\alpha)=\operatorname{supp}(\beta)$. Then we have the following:

(a) $|\operatorname{supp}(\alpha)|=|\operatorname{supp}(\beta)|=2$ and there are $\epsilon, \eta \in \operatorname{supp}(\alpha)=\operatorname{supp}(\beta)$ such that $\operatorname{sgn}(\epsilon ; \alpha)=-\operatorname{sgn}(\epsilon ; \beta)$ and $\operatorname{sgn}(\eta ; \alpha)=\operatorname{sgn}(\eta ; \beta)$.

(b) Keep the same notation as in part (a),

(i) $\epsilon \notin \operatorname{supp}(\gamma)$ for all $\gamma \in B \backslash\{\alpha, \beta\}$; in particular, for $\gamma \in B \backslash\{\alpha, \beta\}$, $(\alpha, \gamma)_{*} \neq 0$ if and only if $(\beta, \gamma)_{*} \neq 0$.

(ii) If $(\cdot, \cdot)_{*}$ is nondegenerate on $\operatorname{span}_{\mathbb{R}} B$, then there is at most one element $\gamma$ of $B \backslash\{\alpha, \beta\}$ with $\eta \in \operatorname{supp}(\gamma)$.

(iii) If $B$ is a base of $R$ and $\eta \in \operatorname{supp}(\theta)$ for some long root $\theta$ of $B$, then for all $\gamma \in B \backslash\{\alpha, \beta, \theta\}, \eta \notin \operatorname{supp}(\gamma)$.

Proof. (a) follows from Fact 1.

(b)(i) Choose $p, q \in \mathbb{Z}$ and $r_{1}, r_{2}, s_{1}, s_{2} \in\{ \pm 1\}$ such that $\alpha=r_{1} \epsilon+r_{2} \eta+p \delta$ and $\beta=s_{1} \epsilon+s_{2} \eta+q \delta$. Without loss of generality, we assume

$$
r_{1}=-s_{1} \quad \text { and } \quad r_{2}=s_{2} \text {. }
$$

We claim that $\epsilon \notin \operatorname{supp}(\gamma)$ for all $\gamma \in B \backslash\{\alpha, \beta\}$. Suppose to the contrary that there is $\gamma \in B \backslash\{\alpha, \beta\}$ such that $\epsilon \in \operatorname{supp}(\gamma)$.

Case 1. $|\operatorname{supp}(\gamma)|=1$ : In this case, $\gamma=2 r \epsilon+k \delta$ for some $r \in\{ \pm 1\}$ and $k \in \mathbb{Z}$. If $r=r_{1}$, then $\alpha-\gamma \in R \cap(B-B)$ which is a contradiction as $B$ is a subset of a base of $R$. Also if $r=-r_{1}=s_{1}$, then $\beta-\gamma \in R \cap(B-B)$ which is again a contradiction.

Case 2. $|\operatorname{supp}(\gamma)|=2$ : Suppose that $\operatorname{supp}(\gamma)=\{\epsilon, \zeta\}$. So $\gamma=r \epsilon+s \zeta+k \delta$ for some $r, s \in\{ \pm 1\}$ and $k \in \mathbb{Z}$. We first assume $r=r_{1}$. If $\zeta \neq \eta$, then we have $\gamma-\alpha=s \zeta-r_{2} \eta+(k-p) \delta \in R \cap(B-B)$ which is a contradiction. Also if $\zeta=\eta$, two cases can happen: $r_{2}=s_{2}=s$ or $r_{2}=s_{2}=-s$. In the former case, we have $(k-p) \delta=\gamma-\alpha \in R \cap(B-B)$ which is a contradiction and in the latter case, we have $s_{1} \epsilon+s \eta+(-p+q+k) \delta=-\alpha+\beta+\gamma$ which is an element of $R$ written as a linear combination of the elements of a base of $R$ with coefficients of opposite sign, a contradiction. Finally, if $r \neq r_{1}$, then $r=s_{1}$ and the same argument as above with changing the role of $\alpha$ and $\beta$ gives a contradiction. This completes the proof.

(b)(ii) Assume $\gamma_{1}$ and $\gamma_{2}$ are two distinct elements of $B \backslash\{\alpha, \beta\}$ with $\eta \in$ $\operatorname{supp}\left(\gamma_{1}\right) \cap \operatorname{supp}\left(\gamma_{2}\right)$. Since $\beta-\gamma_{1}, \beta-\gamma_{2} \notin R$, using part (b)(i) together with Fact 2, we have

$$
\operatorname{sgn}\left(\eta ; \gamma_{1}\right)=-\operatorname{sgn}(\eta ; \beta) \quad \text { and } \operatorname{sgn}\left(\eta ; \gamma_{2}\right)=-\operatorname{sgn}(\eta ; \beta) .
$$

If $\operatorname{supp}\left(\gamma_{1}\right) \neq \operatorname{supp}\left(\gamma_{2}\right)$, then (2.10) implies that $\gamma_{1}-\gamma_{2} \in R$ which is a contradiction. So $\operatorname{supp}\left(\gamma_{1}\right)=\operatorname{supp}\left(\gamma_{2}\right)$. Suppose $\operatorname{supp}\left(\gamma_{1}\right)=\operatorname{supp}\left(\gamma_{2}\right)=\{\eta, \xi\}$. If $\operatorname{sgn}\left(\xi ; \gamma_{1}\right)=$ 
$\operatorname{sgn}\left(\xi ; \gamma_{2}\right)$, (2.10) implies that $\gamma_{1}-\gamma_{2} \in \mathbb{Z} \delta \subseteq R \cap(B-B)$ which is a contradiction. Therefore, $\operatorname{sgn}\left(\xi ; \gamma_{1}\right)=-\operatorname{sgn}\left(\xi ; \gamma_{2}\right)$, so we have

$$
\beta+\gamma_{1}+\gamma_{2}+\alpha \in \mathbb{Z} \delta \cap \operatorname{span}_{\mathbb{Z}} B .
$$

This is a contradiction as $(\cdot, \cdot)_{*}$ is nondegenerate on $\operatorname{span}_{\mathbb{R}} B$. This completes the proof.

(b)(iii) To the contrary, assume $\eta \in \operatorname{supp}(\gamma)$ for some $\gamma \in B \backslash\{\alpha, \beta, \theta\}$. We first mention that using Lemma 2.10, $\operatorname{supp}(\theta) \neq \operatorname{supp}(\gamma)$. Since $\gamma-\theta, \theta-\beta, \alpha-\gamma \notin R$ and $\epsilon \notin \operatorname{supp}(\gamma) \cup \operatorname{supp}(\theta)$ (see part (b)(i)), we have

$$
\operatorname{sgn}(\eta ; \gamma) \stackrel{\text { Fcat }^{2}}{=}-\operatorname{sgn}(\eta ; \alpha) \stackrel{\text { part (a) }}{\underline{=}}-\operatorname{sgn}(\eta ; \beta) \stackrel{\text { Fcat }^{2}}{\operatorname{sgn}(\eta ; \theta)} \stackrel{\text { Fcat }}{2}^{2}-\operatorname{sgn}(\eta ; \gamma)
$$

which is a contradiction.

In what follows we shall show that up to \pm 1 -multiple, the bases introduced in Proposition 2.9 are the only bases of $R$ of type $A(2 m-1,2 n-1)^{(2)},(m, n) \neq(1,1)$.

Definition 2.14. Recall $\theta_{i}$ 's and $\Pi$ from Theorem 1.1 and assume $\operatorname{supp}\left(\theta_{i}\right)=\left\{v_{i}\right\}$. We say $\Pi$ is fine if $\operatorname{sgn}\left(v_{i} ; \theta_{i}\right)=1$.

From now on, we denote the bases introduced in the last four rows of Table 2 respectively by $B^{1}, B^{2}, B^{3}$ and $B^{4}$.

Lemma 2.15. If $\Pi$ is a base of the from $B^{i}(1 \leq i \leq 4)$, then there is $w$ belonging to the quasi-Weyl group $W$ of $R$ such that $w\left(B^{i}\right)$ is a fine base of the from $B^{i}$.

Proof. Suppose $\theta_{i}$ 's are as in Theorem 1.1 and assume $\operatorname{supp}\left(\theta_{i}\right)=\left\{v_{i}\right\}$. For $1 \leq t \leq \ell$, set

$$
r_{t}:= \begin{cases}r_{2 v_{t}} & \text { if } v_{t} \in\left\{\delta_{i} \mid 1 \leq i \leq n\right\} \text { and } \operatorname{sgn}\left(v_{t} ; \theta_{t}\right)=-1, \\ r_{2 v_{t}+\delta} & \text { if } v_{t} \in\left\{\epsilon_{i} \mid 1 \leq i \leq m\right\} \text { and } \operatorname{sgn}\left(v_{t} ; \theta_{t}\right)=-1, \\ \text { id } & \text { otherwise. }\end{cases}
$$

Then $r_{\ell} \cdots r_{1}(R) \subseteq R$; in particular, $\Pi^{\prime}:=r_{1} \cdots r_{\ell}(\Pi)$ is a base of $R$; see Lemma 2.4 and Corollary 2.5. Moreover, $\Pi^{\prime}$ is of the form $B^{i}$. Setting $\theta_{i}^{\prime}:=r_{1} \cdots r_{\ell}\left(\theta_{i}\right)$, we have $\operatorname{supp}\left(\theta_{i}^{\prime}\right)=\left\{v_{i}\right\}$ with $\operatorname{sgn}\left(v_{i} ; \theta_{i}^{\prime}\right)=1$. This completes the proof.

Definition 2.16. Recall $\theta_{i}$ 's and $k_{i}$ 's from Theorem 1.1 and assume $\Pi$ is fine of the form $B^{j}$ for some $1 \leq j \leq 4$. Suppose for each $1 \leq i \leq \ell, \operatorname{supp}\left(\theta_{i}\right)=\left\{v_{i}\right\}$. In this case, we denote $\Pi$ by $\left(j ; v_{1}, \ldots, v_{\ell} ; k_{1}, \ldots, k_{\ell}\right)$.

(i) We say an element $w$ of the quasi-Weyl group $W$ of $R$ preserves the nature of $\Pi$ if $w(\Pi)$ is a base of $R$ of the from $B^{j}$ with $\operatorname{supp}\left(\theta_{i}\right)=\operatorname{supp}\left(w\left(\theta_{i}\right)\right)$ for all $i$.

(ii) We say $\Pi$ is $t$-admissible $(1 \leq t \leq \ell)$ if $k_{1}=\cdots=k_{t}=0$; in this case, we denote $\Pi$ by $\left(j ; v_{1}, \ldots, v_{\ell} ; k_{t+1}, \ldots, k_{\ell}\right)$.

Lemma 2.17. (i) Suppose $B=\left(j ; v_{1}, \ldots, v_{\ell} ; k_{t+1}, \ldots, k_{\ell}\right)$ is a $t$-admissible base for some $t \in\{1, \ldots, \ell-2\}$. Then there is $w \in W$ such that $w$ preserves the nature of $\Pi$ and $w(B)$ is $(t+1)$-admissible.

(ii) If $2 \leq j \leq 4$ and $B=\left(j ; v_{1}, \ldots, v_{\ell} ; k_{2}, \ldots, k_{\ell}\right)$ is a 1-admissible base, then there is $w \in W$ such that $w$ preserves the nature of $\Pi$ and $w(B)$ is $\ell$-admissible. 
Proof. (i) Set

$$
w:=r_{v_{t+1}-v_{t+2}} r_{v_{t+1}-v_{t+2}+k_{t+1} \delta}
$$

then by Corollary 2.5, $w(B)$ is a base of $R$. Moreover,

$w\left(v_{t+1}\right)=v_{t+1}-k_{t+1} \delta, w\left(v_{t+2}\right)=v_{t+2}+k_{t+1} \delta \quad$ and $\quad w\left(v_{i}\right)=v_{i} \quad(i \neq t+1, t+2)$;

in particular,

$$
w\left(\theta_{i}\right)=v_{i} \quad(1 \leq i \leq t+1) \quad \text { and } \quad \operatorname{supp}\left(w\left(\theta_{r}\right)\right)=\operatorname{supp}\left(\theta_{r}\right) \quad(1 \leq r \leq \ell) .
$$

This completes the proof.

(ii) It follows from (i) together with an induction process that there is $w \in W$ such that $w$ preserves the nature of $B$ and $B^{\prime}:=w(B)$ is an $(\ell-1)$-admissible base. So $B^{\prime}=\left(j ; v_{1}, \ldots, v_{\ell} ; k_{\ell}^{\prime}\right)$. We continue the proof in the following cases:

- $\boldsymbol{j}=\mathbf{2}$. In this case, we have $v_{\ell} \in\left\{\delta_{p} \mid 1 \leq p \leq n\right\}$ and

$$
B^{\prime}=\left\{-2 v_{1}, v_{i}-v_{i+1}, v_{\ell-1}-v_{\ell}-k_{\ell}^{\prime} \delta, v_{\ell-1}+v_{\ell}+\left(k_{\ell}^{\prime}+1\right) \delta \mid 1 \leq i \leq \ell-2\right\} .
$$

If $k_{\ell}^{\prime}$ is odd, set

$$
w_{0}:=r_{2 v_{\ell}+\left(k_{\ell}^{\prime}+1\right) \delta}
$$

then using Lemma 2.4 and Corollary 2.5, $w_{0}\left(B^{\prime}\right)$ is a base of $R$,

$$
w_{0}\left(v_{\ell}\right)=-v_{\ell}-k_{\ell}^{\prime} \delta \quad \text { and } \quad w_{0}\left(v_{i}\right)=v_{i} \quad(1 \leq i \leq \ell-1) ;
$$

in particular,

$$
\begin{aligned}
w_{0}\left(\theta_{\ell-1}-\theta_{\ell}\right) & =w_{0}\left(v_{\ell-1}-v_{\ell}-k_{\ell}^{\prime} \delta\right)=v_{\ell-1}+v_{\ell}+\delta \\
w_{0}\left(\theta_{\ell-1}+\theta_{\ell}+\delta\right) & =w_{0}\left(v_{\ell-1}+v_{\ell}+\left(k_{\ell}^{\prime}+1\right) d\right)=v_{\ell-1}-v_{\ell}
\end{aligned}
$$

and we are done in this case. Also if $k_{\ell}^{\prime}$ is even, we set

$$
w_{0}:=r_{2 v_{\ell}} r_{2 v_{\ell}+k_{\ell}^{\prime} \delta}
$$

We have again that $w_{0}\left(B^{\prime}\right)$ is a base of $R$ and moreover, we have

$$
w_{0}\left(v_{\ell}\right)=v_{\ell}-k_{\ell}^{\prime} \delta \quad \text { and } \quad w_{0}\left(v_{i}\right)=v_{i} \quad(1 \leq i \leq \ell-1) ;
$$

in particular,

$$
\begin{aligned}
w_{0}\left(\theta_{\ell-1}-\theta_{\ell}\right) & =w_{0}\left(v_{\ell-1}-v_{\ell}-k_{\ell}^{\prime} \delta\right)=v_{\ell-1}-v_{\ell} \\
w_{0}\left(\theta_{\ell-1}+\theta_{\ell}+\delta\right) & =w_{0}\left(v_{\ell-1}+v_{\ell}+\left(k_{\ell}^{\prime}+1\right) d\right)=v_{\ell-1}+v_{\ell}+\delta .
\end{aligned}
$$

This completes the proof for $j=2$..

- $j=3$. In this case, we have $v_{\ell} \in\left\{\epsilon_{i} \mid 1 \leq i \leq m\right\}$ and

$$
B^{\prime}=\left\{-2 v_{1}+\delta, v_{i}-v_{i+1}, v_{\ell-1}-v_{\ell}-k_{\ell}^{\prime} \delta, v_{\ell-1}+v_{\ell}+k_{\ell}^{\prime} \delta \mid 1 \leq i \leq \ell-2\right\} .
$$

Set

$$
w_{0}:= \begin{cases}r_{2 v_{\ell}+k_{\ell}^{\prime} \delta} & \text { if } k_{\ell}^{\prime} \text { is odd, } \\ r_{2 v_{\ell}+\delta} r_{2 v_{\ell}+\left(k_{\ell}^{\prime}+1\right) \delta} & \text { if } k_{\ell}^{\prime} \text { is even, }\end{cases}
$$

then as above $w_{0}\left(B^{\prime}\right)$ is an $\ell$-admissible base.

- $j=4$. In this case, we have $v_{1} \in\left\{\delta_{p} \mid 1 \leq p \leq m\right\}, v_{\ell} \in\left\{\epsilon_{i} \mid 1 \leq i \leq m\right\}$ and

$$
B^{\prime}=\left\{-2 v_{1}, v_{i}-v_{i+1}, v_{\ell-1}-v_{\ell}-k_{\ell}^{\prime} \delta, 2 v_{\ell}+2 k_{\ell}^{\prime} \delta+\delta \mid 1 \leq i \leq \ell-2\right\} .
$$

Setting

we get that

$$
w_{0}:= \begin{cases}r_{2 v_{1}} r_{v_{1}-v_{\ell}} r_{v_{1}+v_{\ell}} r_{2 v_{\ell}+k_{\ell}^{\prime} \delta} & \text { if } k_{\ell}^{\prime} \text { is odd, } \\ r_{2 v_{\ell}+\delta} r_{2 v_{\ell}+\left(k_{\ell}^{\prime}+1\right) \delta} & \text { if } k_{\ell}^{\prime} \text { is even, }\end{cases}
$$

$$
w_{0}\left(v_{\ell}\right)=v_{\ell}-k_{\ell}^{\prime} \delta \quad \text { and } \quad w_{0}\left(v_{i}\right)=v_{i} \quad(1 \leq i \leq \ell-1)
$$


and that $w_{0}\left(B^{\prime}\right)$ is an $\ell$-admissible base; see Lemma 2.4 and Corollary 2.5 .

Corollary 2.18. If $B$ and $B^{\prime}$ are two bases of $R$ of the form $B^{j}$ for some $2 \leq j \leq 4$, then $B$ and $B^{\prime}$ are conjugate under the quasi-Weyl group.

Proof. Using Lemma 2.17(ii), without loss of generality, we assume $B$ and $B^{\prime}$ are $\ell$-admissible. So there are distinct elements $v_{1}, \ldots, v_{\ell}$ as well as distinct elements $v_{1}^{\prime}, \ldots, v_{\ell}^{\prime}$ of $\left\{\epsilon_{i}, \delta_{p} \mid 1 \leq i \leq m, 1 \leq p \leq n\right\}$ such that

$$
B=\left(j ; v_{1}, \ldots, v_{\ell}\right) \quad \text { and } \quad B^{\prime}=\left(j ; v_{1}^{\prime}, \ldots, v_{\ell}^{\prime}\right) \text {. }
$$

Case 1. $v_{1}=v_{1}^{\prime}$ and $v_{\ell}=v_{\ell}^{\prime}$ : Define

$$
w_{2}:=\left\{\begin{array}{ll}
\text { id } & \text { if } v_{2}=v_{2}^{\prime} \\
r_{v_{2}-v_{2}^{\prime}} & \text { if } v_{2} \neq v_{2}^{\prime}
\end{array} .\right.
$$

We next assume $w_{2}, \ldots, w_{t}(1 \leq t \leq \ell-2)$ have been defined and set

$$
\alpha_{t+1}:=w_{t} \cdots w_{2}\left(v_{t+1}^{\prime}\right) \quad \text { and } \quad w_{t+1}:= \begin{cases}\text { id } & \text { if } v_{t+1}=\alpha_{t+1} \\ r_{v_{t+1}-\alpha_{t+1}} & \text { if } v_{t+1} \neq \alpha_{t+1}\end{cases}
$$

We claim that

$$
w_{t} \cdots w_{2}\left(v_{1}^{\prime}\right)=v_{1} \quad \text { and } \quad w_{t} \cdots w_{2}\left(v_{j}^{\prime}\right)=v_{j} \quad(2 \leq j \leq t \leq \ell) .
$$

We use an induction process to do this; by definition, we have

$$
w_{2}\left(v_{1}^{\prime}\right)=w_{2}\left(v_{1}\right)=v_{1} \quad \text { and } \quad w_{2}\left(v_{2}^{\prime}\right)=v_{2} .
$$

Next assume $2 \leq t \leq \ell-1$ and that $w_{t} \cdots w_{2}\left(v_{j}^{\prime}\right)=v_{j}$ for $1 \leq j \leq t$. We shall show

$$
w_{t+1} \cdots w_{2}\left(v_{j}^{\prime}\right)=v_{j} \quad(1 \leq j \leq t+1) .
$$

Suppose $1 \leq j \leq t$. Since $v_{1}, \ldots, v_{\ell}$ are distinct, we have $v_{j} \neq v_{t+1}$. Also $v_{j} \neq \alpha_{t+1}$ as otherwise by the induction hypothesis, we have

$$
w_{t} \cdots w_{2}\left(v_{t+1}^{\prime}\right)=\alpha_{t+1}=v_{j}=w_{t} \cdots w_{2}\left(v_{j}^{\prime}\right)
$$

which in turn implies that $v_{t+1}^{\prime}=v_{j}^{\prime}$, a contradiction. Therefore, $w_{t+1}\left(v_{j}\right)=v_{j}$ and so we have using induction hypothesis that

$$
w_{t+1} w_{t} \cdots w_{2}\left(v_{j}^{\prime}\right)= \begin{cases}w_{t+1}\left(v_{j}\right)=v_{j} & 1 \leq j \leq t \\ w_{t+1}\left(\alpha_{t+1}\right)=v_{t+1}=v_{j} & j=t+1 .\end{cases}
$$

This completes the proof of (2.11). Now set $w:=w_{\ell} \cdots w_{2}$, we have $w\left(B^{\prime}\right)=B$ as we desired.

Case 2. General Case: We recall Lemma 2.4 and Corollary 2.5 and set

$$
\omega_{1}:=\left\{\begin{array}{ll}
\text { id } & \text { if } v_{1}=v_{1}^{\prime} \\
r_{v_{1}-v_{1}^{\prime}} & \text { if } v_{1} \neq v_{1}^{\prime} .
\end{array} \text { and } \Pi:=\omega_{1}\left(B^{\prime}\right) .\right.
$$

Then for $v_{i}^{\prime \prime}:=\omega_{1}\left(v_{i}^{\prime}\right) \in\left\{\epsilon_{i}, \delta_{p} \mid 1 \leq i \leq m, 1 \leq p \leq n\right\}$, we have

$$
\Pi=\left(j ; v_{1}^{\prime \prime}, \ldots, v_{\ell}^{\prime \prime}\right) \text { with } v_{1}^{\prime \prime}=v_{1} \text {. }
$$

Next we set

$$
\omega_{2}:=\left\{\begin{array}{ll}
\text { id } & \text { if } v_{\ell}=v_{\ell}^{\prime \prime} \\
r_{v_{\ell}-v_{\ell}^{\prime \prime}} & \text { if } v_{\ell} \neq v_{\ell}^{\prime \prime}
\end{array} \text { and } \Pi^{\prime}:=\omega_{2}(\Pi) .\right.
$$

Then for $v_{i}^{\prime \prime \prime}:=\omega_{2}\left(v_{i}^{\prime \prime}\right) \in\left\{\epsilon_{i}, \delta_{p} \mid 1 \leq i \leq m, 1 \leq p \leq n\right\}$, we have

$$
\Pi^{\prime}=\left(j ; v_{1}^{\prime \prime \prime}, \ldots, v_{\ell}^{\prime \prime \prime}\right) \text { with } v_{1}^{\prime \prime \prime}=v_{1} \text { and } v_{\ell}^{\prime \prime \prime}=v_{\ell} .
$$

So we are done using Case 1. 
Lemma 2.19. Suppose that $B$ is a nonempty subset of $R^{\times}$with $\left|B \cap R_{l g}\right| \leq 1$; see (2.7). Assume $W_{B}$ is the subgroup of quasi-Weyl group $W$ of $R$ generated by the quasi-reflections based on the elements of $B$. Set $S:=W_{B} B$ and assume $\left(\operatorname{span}_{\mathbb{R}} S,(\cdot, \cdot)_{*}, S\right)$ is an irreducible finite root system with base $B$, then it is of one of types $A_{\mathfrak{r}}(\mathfrak{r} \geq 1), D_{\mathfrak{r}}(\mathfrak{r} \geq 4)$ or $C_{\mathfrak{r}}(\mathfrak{r} \geq 2)$.

Proof. If $B \cap R_{l g}=\emptyset$, then $S \subseteq R$. So using Corollary 2.12, $S$ is not of type $E_{6,7,8}$. Since bases of finite root systems of types $F_{4}$ and $B_{\mathfrak{r}}(\mathfrak{r} \geq 3)$ contain at least two long roots and $B$ contains at most one long root, we get that $S$ is not neither of type $B_{\mathfrak{r}}(\mathfrak{r} \geq 3)$ nor of type $F_{4}$. Also as the ratio of the lengths of the roots in $S$ is $1,1 / 2$ or $2, S$ is not also of type $G_{2}$.

Lemma 2.20. Suppose that $\Pi$ is a base of $R$ and $\alpha \in \Pi$ is a long root. Suppose $B \subseteq \Pi \backslash\{\alpha\}$ is such that for $S:=W_{B} B,\left(\operatorname{span}_{\mathbb{R}} S,(\cdot, \cdot)_{*}, S\right)$ is an irreducible finite root system of rank $\mathfrak{r}$ and $B$ is a base of $S$.

(i) If $S$ is of type $D_{\mathfrak{r}}(\mathfrak{r} \geq 4)$, then there are distinct elements $\dot{\zeta}_{1}, \ldots, \dot{\zeta}_{\mathfrak{r}} \in$ $\left\{\epsilon_{t}, \delta_{p} \mid 1 \leq t \leq m, 1 \leq p \leq n\right\}$ and $\alpha_{1}, \ldots, \alpha_{\mathfrak{r}} \in R$ with

- $\operatorname{supp}\left(\alpha_{i}\right)=\left\{\dot{\zeta}_{i}, \dot{\zeta}_{i+1}\right\}$ for $1 \leq i \leq \mathfrak{r}-1$,

- $\operatorname{supp}\left(\alpha_{\mathfrak{r}-1}\right)=\operatorname{supp}\left(\alpha_{\mathfrak{r}}\right)$ and $\left(\alpha_{\mathfrak{r}-1}, \alpha_{\mathfrak{r}}\right)_{*}=0$,

- for $1 \leq i \leq \mathfrak{r}-1, \operatorname{sgn}\left(\dot{\zeta}_{i+1} ; \alpha_{i}\right)=-\operatorname{sgn}\left(\dot{\zeta}_{i+1} ; \alpha_{i+1}\right)$,

- $\operatorname{sgn}\left(\dot{\zeta}_{\mathfrak{r}-1} ; \alpha_{\mathfrak{r}-1}\right)=\operatorname{sgn}\left(\dot{\zeta}_{\mathfrak{r}-1} ; \alpha_{\mathfrak{r}}\right)$,

- $B=\left\{\alpha_{1}, \ldots, \alpha_{\mathrm{r}}\right\}$.

(ii) If $S$ is of type $C_{\mathfrak{r}}(\mathfrak{r} \geq 2)$, then there are distinct elements $\dot{\zeta}_{1}, \ldots, \dot{\zeta}_{\mathfrak{r}} \in$ $\left\{\epsilon_{t}, \delta_{p} \mid 1 \leq t \leq m, 1 \leq p \leq n\right\}$ and $\alpha_{1}, \ldots, \alpha_{\mathfrak{r}} \in R$ with

- $\operatorname{supp}\left(\alpha_{i}\right)=\left\{\dot{\zeta}_{i}, \dot{\zeta}_{i+1}\right\}$ for $1 \leq i \leq \mathfrak{r}-1$,

- $\operatorname{supp}\left(\alpha_{\mathfrak{r}}\right)=\left\{\dot{\zeta}_{\mathfrak{r}}\right\}$,

- for $1 \leq i \leq \mathfrak{r}, \operatorname{sgn}\left(\dot{\zeta}_{i+1} ; \alpha_{i}\right)=-\operatorname{sgn}\left(\dot{\zeta}_{i+1} ; \alpha_{i+1}\right)$,

- $B=\left\{\alpha_{1}, \ldots, \alpha_{\mathfrak{r}}\right\}$.

(iii) If $S$ is of type $A_{\mathfrak{r}}$ for some $\mathfrak{r} \neq 1,3$ or $S \cap R_{l g}=\emptyset$ and $\mathfrak{r}=1$, then there are distinct elements $\dot{\zeta}_{1}, \ldots, \dot{\zeta}_{\mathfrak{r}+1} \in\left\{\epsilon_{t}, \delta_{p} \mid 1 \leq t \leq m, 1 \leq p \leq n\right\}$ and $\left\{\alpha_{1}, \ldots, \alpha_{\mathfrak{r}}\right\}$ with

- $\operatorname{supp}\left(\alpha_{i}\right)=\left\{\dot{\zeta}_{i}, \dot{\zeta}_{i+1}\right\}$ for $1 \leq i \leq \mathfrak{r}$,

- for $1 \leq i \leq \mathfrak{r}, \operatorname{sgn}\left(\dot{\zeta}_{i+1} ; \alpha_{i}\right)=-\operatorname{sgn}\left(\dot{\zeta}_{i+1} ; \alpha_{i+1}\right)$,

- $B=\left\{\alpha_{1}, \ldots, \alpha_{\mathrm{r}}\right\}$.

(iv) If $S$ is of type $A_{3}$, then one of the following happens:

(a) There are $\alpha_{1}, \alpha_{2}, \alpha_{3} \in R$ and distinct elements $\dot{\zeta}_{1}, \dot{\zeta}_{2}, \dot{\zeta}_{3}, \dot{\zeta}_{4} \in\left\{\epsilon_{t}, \delta_{p} \mid\right.$ $1 \leq t \leq m, 1 \leq p \leq n\}$ with

- $\operatorname{supp}\left(\alpha_{i}\right)=\left\{\dot{\zeta}_{i}, \dot{\zeta}_{i+1}\right\}$ for $1 \leq i \leq 3$,

- for $1 \leq i \leq 3, \operatorname{sgn}\left(\dot{\zeta}_{i+1} ; \alpha_{i}\right)=-\operatorname{sgn}\left(\dot{\zeta}_{i+1} ; \alpha_{i+1}\right)$,

- $B=\left\{\alpha_{1}, \alpha_{2}, \alpha_{3}\right\}$.

(b) There are distinct elements $\dot{\zeta}_{1}, \dot{\zeta}_{2}, \dot{\zeta}_{3} \in\left\{\epsilon_{t}, \delta_{p} \mid 1 \leq t \leq m, 1 \leq p \leq n\right\}$ and $\alpha_{1}, \alpha_{2}, \alpha_{3} \in R$ with

- $\operatorname{supp}\left(\alpha_{i}\right)=\left\{\dot{\zeta}_{i}, \dot{\zeta}_{i+1}\right\}$ for $1 \leq i \leq 2$,

- $\operatorname{supp}\left(\alpha_{2}\right)=\operatorname{supp}\left(\alpha_{3}\right)$ and $\left(\alpha_{2}, \alpha_{3}\right)_{*}=0$,

- for $1 \leq i \leq 2, \operatorname{sgn}\left(\dot{\zeta}_{i+1} ; \alpha_{i}\right)=-\operatorname{sgn}\left(\dot{\zeta}_{i+1} ; \alpha_{i+1}\right)$,

- $\operatorname{sgn}\left(\dot{\zeta}_{2} ; \alpha_{2}\right)=\operatorname{sgn}\left(\dot{\zeta}_{2} ; \alpha_{3}\right)$,

- $B=\left\{\alpha_{1}, \alpha_{2}, \alpha_{3}\right\}$.

Proof. (i) By our assumption, $B$ is a base of a finite root system of type $D_{\mathfrak{r}}$. Suppose $B=\left\{\alpha_{1}, \ldots, \alpha_{\mathfrak{r}}\right\}$ with the corresponding Dynkin diagram 


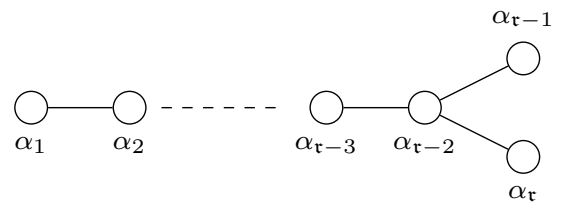

and note that by Lemma 2.10, for each $i,\left|\operatorname{supp}\left(\alpha_{i}\right)\right|=2$.

Convention. If $\mathfrak{r}=4$, changing the indices if necessary and using Lemma 2.11, we assume $\operatorname{supp}\left(\alpha_{\mathfrak{r}}\right)=\operatorname{supp}\left(\alpha_{\mathfrak{r}-1}\right)$.

Step 1. We have $\operatorname{supp}\left(\alpha_{\mathfrak{r}}\right)=\operatorname{supp}\left(\alpha_{\mathfrak{r}-1}\right)$ : It follows from the above convention, Lemmas 2.11 and 2.13(b)(i) together with the fact that if $\mathfrak{r}>4$, we have $\left(\alpha_{\mathfrak{r}-4}, \alpha_{\mathfrak{r}}\right)_{*},\left(\alpha_{\mathfrak{r}-4}, \alpha_{\mathfrak{r}-1}\right)_{*}=0$ while $\left(\alpha_{\mathfrak{r}-4}, \alpha_{\mathfrak{r}-3}\right)_{*} \neq 0$.

Step 2. For $1 \leq i<j \leq \mathfrak{r}-2$, we have $\operatorname{supp}\left(\alpha_{i}\right) \cap \operatorname{supp}\left(\alpha_{j+1}\right)=\emptyset:$ Suppose to the contrary that $1 \leq i<j \leq \mathfrak{r}-2$ and $\operatorname{supp}\left(\alpha_{i}\right) \cap \operatorname{supp}\left(\alpha_{j+1}\right) \neq \emptyset$. Since $\left(\alpha_{i}, \alpha_{j+1}\right)_{*}=0$, we have $\operatorname{supp}\left(\alpha_{i}\right)=\operatorname{supp}\left(\alpha_{j+1}\right)$ and using Lemma 2.13(b)(i), for $\gamma \in B \backslash\left\{\alpha_{i}, \alpha_{j+1}\right\},\left(\gamma, \alpha_{i}\right)_{*} \neq 0$ if and only if $\left(\gamma, \alpha_{j+1}\right)_{*} \neq 0$. But if $j \neq \mathfrak{r}-2$, $\left(\alpha_{i}, \alpha_{j+2}\right)_{*}=0$ while $\left(\alpha_{j+1}, \alpha_{j+2}\right)_{*} \neq 0$, a contradiction. Also if $j=\mathfrak{r}-2$, using Step 1, we have $\operatorname{supp}\left(\alpha_{i}\right)=\operatorname{supp}\left(\alpha_{j+1}\right)=\operatorname{supp}\left(\alpha_{j+2}\right)$ which contradicts Lemma 2.13(b)(i).

Step 3. For $1 \leq i \leq \mathfrak{r}-2,\left|\operatorname{supp}\left(\alpha_{i}\right) \cap \operatorname{supp}\left(\alpha_{i+1}\right)\right|=1$ : Since $\left(\alpha_{i}, \alpha_{i+1}\right)_{*} \neq$ 0 , we have $\operatorname{supp}\left(\alpha_{i}\right) \cap \operatorname{supp}\left(\alpha_{i+1}\right) \neq \emptyset$. If $\left|\operatorname{supp}\left(\alpha_{i}\right) \cap \operatorname{supp}\left(\alpha_{i+1}\right)\right| \neq 1$, we have $\left|\operatorname{supp}\left(\alpha_{i}\right) \cap \operatorname{supp}\left(\alpha_{i+1}\right)\right|=2$. Suppose $\operatorname{supp}\left(\alpha_{i}\right)=\operatorname{supp}\left(\alpha_{i+1}\right)=\{\zeta, \eta\}$, then since $\left(\alpha_{i}, \alpha_{i+1}\right)_{*} \neq 0$, there is $r \in\{ \pm 1\}$ with $\operatorname{sgn}\left(\zeta ; \alpha_{i}\right)=r \operatorname{sgn}\left(\zeta ; \alpha_{i+1}\right)$ and $\operatorname{sgn}\left(\eta ; \alpha_{i}\right)=$ $r \operatorname{sgn}\left(\eta ; \alpha_{i+1}\right)$. But this implies that $\alpha_{i}-r \alpha_{i+1} \in \mathbb{Z} \delta$. Since $(\cdot, \cdot)_{*}$ is nondegenerate on $\operatorname{span}_{\mathbb{R}} B$, we have $\alpha_{i}-r \alpha_{i+1}=0$ which is a contradiction.

Step 4. Use Steps 1-3 to pick distinct elements $\dot{\zeta}_{i} \in\left\{\epsilon_{t}, \delta_{p} \mid 1 \leq t \leq m, 1 \leq j \leq n\right\}$ $(1 \leq i \leq \mathfrak{r})$ with $\dot{\zeta}_{i} \in \operatorname{supp}\left(\alpha_{i}\right)$ and for $1 \leq i \leq \mathfrak{r}-2, \operatorname{supp}\left(\alpha_{i}\right) \cap \operatorname{supp}\left(\alpha_{i+1}\right)=\left\{\dot{\zeta}_{i+1}\right\}$. For $1 \leq i \leq \mathfrak{r}-2, \operatorname{sgn}\left(\dot{\zeta}_{i+1} ; \alpha_{i}\right)=-\operatorname{sgn}\left(\dot{\zeta}_{i+1} ; \alpha_{i+1}\right)$ as otherwise, $\alpha_{i}-\alpha_{i+1} \in R$ which is a contradiction (see Fact 2). Similarly, $\operatorname{sgn}\left(\dot{\zeta}_{\mathfrak{r}-1} ; \alpha_{\mathfrak{r}-2}\right)=-\operatorname{sgn}\left(\dot{\zeta}_{\mathfrak{r}-1} ; \alpha_{\mathfrak{r}}\right)$. This implies that $\operatorname{sgn}\left(\dot{\zeta}_{\mathfrak{r}-1} ; \alpha_{\mathfrak{r}-1}\right)=\operatorname{sgn}\left(\dot{\zeta}_{\mathfrak{r}-1} ; \alpha_{\mathfrak{r}}\right)$ and $\operatorname{sos} \operatorname{sgn}\left(\dot{\zeta}_{\mathfrak{r}} ; \alpha_{\mathfrak{r}-1}\right)=-\operatorname{sgn}\left(\dot{\zeta}_{\mathfrak{r}} ; \alpha_{\mathfrak{r}}\right)$ (see Fact 1).

(ii) is similarly proved.

(iii),(iv) Suppose $B=\left\{\alpha_{1}, \ldots, \alpha_{\mathfrak{r}}\right\}$ such that the corresponding Dynkin diagram of $S$ is as follows:

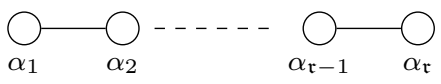

As in Step 3 of the proof of part (i), for $1 \leq i \leq \mathfrak{r}+1$, there is $\dot{\zeta}_{i} \in\left\{\epsilon_{j}, \delta_{p}\right.$ $1 \leq j \leq m, 1 \leq p \leq n\}$ with $\dot{\zeta}_{1} \in \operatorname{supp}\left(\alpha_{1}\right), \dot{\zeta}_{\mathfrak{r}+1} \in \operatorname{supp}\left(\alpha_{\mathfrak{r}}\right)$ and for $1 \leq i \leq$ $\mathfrak{r}-1, \operatorname{supp}\left(\alpha_{i}\right) \cap \operatorname{supp}\left(\alpha_{i+1}\right)=\left\{\dot{\zeta}_{i+1}\right\}$ and as in the proof of Step 4 of part (i), $\operatorname{sgn}\left(\dot{\zeta}_{i+1} ; \alpha_{i}\right)=-\operatorname{sgn}\left(\dot{\zeta}_{i+1} ; \alpha_{i+1}\right)$ for $1 \leq i \leq \mathfrak{r}-1$. This completes the proof if $\dot{\zeta}_{i}$ 's are distinct.

Now assume $\dot{\zeta}_{i}$ 's are not distinct. Then there are $1 \leq i<i+1<j \leq \mathfrak{r}$ with $\operatorname{supp}\left(\alpha_{i}\right) \cap \operatorname{supp}\left(\alpha_{j}\right) \neq \emptyset$. Since $\left(\alpha_{i}, \alpha_{j}\right)_{*}=0$, we have $\operatorname{supp}\left(\alpha_{i}\right)=\operatorname{supp}\left(\alpha_{j}\right)$. Assume $\operatorname{supp}\left(\alpha_{i}\right)=\operatorname{supp}\left(\alpha_{j}\right)=\{\zeta, \eta\}$. Using Lemma 2.13(a),(b)(i), we assume $\operatorname{sgn}\left(\zeta ; \alpha_{i}\right)=-\operatorname{sgn}\left(\zeta ; \alpha_{j}\right), \operatorname{sgn}\left(\eta ; \alpha_{i}\right)=\operatorname{sgn}\left(\eta ; \alpha_{j}\right)$ and

$$
\zeta \notin \operatorname{supp}(\gamma) \quad\left(\gamma \in B \backslash\left\{\alpha_{i}, \alpha_{j}\right\}\right) .
$$


We know $\left(\alpha_{i}, \alpha_{i+1}\right)_{*} \neq 0$ and $\left(\alpha_{j-1}, \alpha_{j}\right)_{*} \neq 0$, so

$$
\eta \in \operatorname{supp}\left(\alpha_{i+1}\right) \cap \operatorname{supp}\left(\alpha_{j-1}\right) .
$$

This together with Lemma 2.13(b)(ii) implies that

$$
i+1=j-1 \text {. }
$$

Now if $i \neq 1$, since $\left(\alpha_{i-1}, \alpha_{i}\right)_{*} \neq 0,(2.12)$ implies that $\eta \in \operatorname{supp}\left(\alpha_{i-1}\right)$ which is a contradiction due to Lemma2.13(b)(ii) and similarly, if $j \neq \mathfrak{r}$, we have $\left(\alpha_{j+1}, \alpha_{j}\right)_{*} \neq$ 0 which is a contradiction as above. So $i=1$ and $j=\mathfrak{r}$; i.e., $\mathfrak{r}=3$ and we are done.

Corollary 2.21. Suppose that $\Pi$ is a base of $R$ and $\alpha \in \Pi$ is a long root. Suppose $B \subseteq \Pi \backslash\{\alpha\}$ such that for $S:=W_{B} B,\left(\operatorname{span}_{\mathbb{R}} S,(\cdot, \cdot)_{*}, S\right)$ is an irreducible finite root system of rank $\mathfrak{r}, B$ is a base of $S$ and $(\alpha, B)_{*} \neq\{0\}$.

(i) If $S$ is of type $D_{\mathfrak{r}}(\mathfrak{r} \geq 4)$, then there are

$$
r \in\{0,1\}, \quad k^{*} \in \mathbb{Z} \quad \text { and } \zeta_{1}, \ldots, \zeta_{\mathfrak{r}} \in\left\{ \pm \epsilon_{t}, \pm \delta_{p} \mid 1 \leq t \leq n, 1 \leq p \leq m\right\}+\mathbb{Z} \delta
$$

with $\operatorname{supp}\left(\zeta_{i}\right)=\left\{\dot{\zeta}_{i}\right\}$ such that $\operatorname{supp}(\alpha)=\left\{\dot{\zeta}_{1}\right\}, \dot{\zeta}_{i}$ 's are distinct,

$$
\alpha=-2 \zeta_{1}+r \delta \quad \text { and } \quad B=\left\{\zeta_{i}-\zeta_{i+1}, \zeta_{\mathfrak{r}-1}+\zeta_{\mathfrak{r}}+k^{*} \delta \mid 1 \leq i \leq \mathfrak{r}-1\right\} .
$$

(ii) If $S$ is of type $C_{\mathfrak{r}}(\mathfrak{r} \geq 2)$, then there are

$r \in\{0,1\}, \quad k^{*} \in \mathbb{Z} \quad$ and $\quad \zeta_{1}, \ldots, \zeta_{\mathfrak{r}} \in\left\{ \pm \epsilon_{t}, \pm \delta_{p} \mid 1 \leq t \leq n, 1 \leq p \leq m\right\}+\mathbb{Z} \delta$ with $\operatorname{supp}\left(\zeta_{i}\right)=\left\{\dot{\zeta}_{i}\right\}$ such that $\operatorname{supp}(\alpha)=\left\{\dot{\zeta}_{1}\right\}, \dot{\zeta}_{i}$ 's are distinct,

$$
\alpha=-2 \zeta_{1}+r \delta \text { and } B=\left\{\zeta_{i}-\zeta_{i+1}, 2 \zeta_{\mathfrak{r}}+k^{*} \delta \mid 1 \leq i \leq \mathfrak{r}\right\} .
$$

(iii) If $S$ is of type $A_{\mathfrak{r}}(\mathfrak{r} \neq 3)$, then there are

$r \in\{0,1\}, \zeta_{1}, \ldots, \zeta_{\mathfrak{r}+1} \in\left\{ \pm \epsilon_{t}, \pm \delta_{p} \mid 1 \leq t \leq n, 1 \leq p \leq m\right\}+\mathbb{Z} \delta$

with $\operatorname{supp}\left(\zeta_{i}\right)=\left\{\dot{\zeta}_{i}\right\}$ such that $\operatorname{supp}(\alpha)=\left\{\dot{\zeta}_{1}\right\}, \dot{\zeta}_{i}$ 's are distinct,

$$
\alpha=-2 \zeta_{1}+r \delta \quad \text { and } B=\left\{\zeta_{i}-\zeta_{i+1} \mid 1 \leq i \leq \mathfrak{r}\right\} .
$$

(iv) If $S$ is of type $A_{3}$, then one of the following happens:

(a) There are

$$
\begin{gathered}
r \in\{0,1\}, k^{*} \in \mathbb{Z} \quad \text { and } \zeta_{1}, \zeta_{2}, \zeta_{3} \in\left\{ \pm \epsilon_{t}, \pm \delta_{p} \mid 1 \leq t \leq n, 1 \leq p \leq m\right\}+\mathbb{Z} \delta \\
\text { with } \operatorname{supp}\left(\zeta_{i}\right)=\left\{\dot{\zeta}_{i}\right\} \text { such that } \operatorname{supp}(\alpha)=\left\{\dot{\zeta}_{1}\right\}, \dot{\zeta}_{i} \text { 's are distinct, } \\
\alpha=-2 \zeta_{1}+r \delta \quad \text { and } B=\left\{\zeta_{1}-\zeta_{2}, \zeta_{2}-\zeta_{3}, \zeta_{2}+\zeta_{3}+k^{*} \delta\right\} .
\end{gathered}
$$

(b) There are

$$
\begin{gathered}
r \in\{0,1\}, \quad \zeta_{1}, \zeta_{2}, \zeta_{3}, \zeta_{4} \in\left\{ \pm \epsilon_{t}, \pm \delta_{p} \mid 1 \leq t \leq n, 1 \leq p \leq m\right\}+\mathbb{Z} \delta \\
\text { with } \operatorname{supp}\left(\zeta_{i}\right)=\left\{\dot{\zeta}_{i}\right\} \text { such that } \operatorname{supp}(\alpha)=\left\{\dot{\zeta}_{1}\right\}, \dot{\zeta}_{i} \text { 's are distinct, } \\
\alpha=-2 \zeta_{1}+r \delta \quad \text { and } B=\left\{\zeta_{i}-\zeta_{i+1} \mid 1 \leq i \leq 3\right\} .
\end{gathered}
$$

Proof. (i) By Lemma 2.20(i), there are distinct elements $\dot{\zeta}_{1}, \ldots, \dot{\zeta}_{\mathfrak{r}} \in\left\{\epsilon_{i}, \delta_{p} \mid\right.$ $1 \leq i \leq m, 1 \leq p \leq n\}, k_{1}, \ldots, k_{\mathfrak{r}} \in \mathbb{Z}$ and $\alpha_{1}, \ldots, \alpha_{\mathfrak{r}} \in R$ such that $\operatorname{supp}\left(\alpha_{i}\right)=$ $\left\{\dot{\zeta}_{i}, \dot{\zeta}_{i+1}\right\}(1 \leq i \leq \mathfrak{r}-1)$ and $\operatorname{supp}\left(\alpha_{\mathfrak{r}}\right)=\left\{\dot{\zeta}_{\mathfrak{r}-1}, \dot{\zeta}_{\mathfrak{r}}\right\}$. Also for

$$
\begin{gathered}
\eta_{i}:=\operatorname{sgn}\left(\dot{\zeta}_{i} ; \alpha_{i}\right) \dot{\zeta}_{i}, \\
B=\left\{\alpha_{i}:=\eta_{i}-\eta_{i+1}+k_{i} \delta, \alpha_{\mathfrak{r}}:=\eta_{\mathfrak{r}-1}+\eta_{\mathfrak{r}}+k_{\mathfrak{r}} \delta \mid 1 \leq i \leq \mathfrak{r}-1\right\} .
\end{gathered}
$$


Since $(\alpha, B)_{*} \neq\{0\}, \operatorname{supp}(\alpha)=\left\{\dot{\zeta}_{j}\right\}$ for some $1 \leq j \leq \mathfrak{r}$. Suppose $j \neq 1$, then since $\alpha-\alpha_{j-1}, \alpha-\alpha_{j} \notin R$, using Fact 2 , we have $\operatorname{sgn}\left(\dot{\zeta}_{j} ; \alpha\right)=-\operatorname{sgn}\left(\dot{\zeta}_{j} ; \alpha_{j}\right)$ and $\operatorname{sgn}\left(\dot{\zeta}_{j} ; \alpha\right)=-\operatorname{sgn}\left(\dot{\zeta}_{j} ; \alpha_{j-1}\right)$ which is a contradiction. So $\operatorname{supp}(\alpha)=\left\{\dot{\zeta}_{1}\right\}$ and as $\alpha-\alpha_{1} \notin R, \operatorname{sgn}\left(\dot{\zeta}_{1} ; \alpha\right)=-\operatorname{sgn}\left(\dot{\zeta}_{1} ; \alpha_{1}\right)$. So $\alpha=-2 \eta_{1}+2 k_{0} \delta+r \delta$, for some $k_{0} \in \mathbb{Z}$ and $r \in\{0,1\}$. Set

$$
k^{*}:=k_{\mathfrak{r}}+k_{\mathfrak{r}-1}+2 \sum_{j=0}^{\mathfrak{r}-2} k_{j} \quad \text { and } \quad \zeta_{i}:=\eta_{i}-\sum_{j=0}^{i-1} k_{j} \delta \quad(1 \leq i \leq \mathfrak{r}) .
$$

Then we have $B=\left\{\alpha_{i}=\zeta_{i}-\zeta_{i+1}, \alpha_{\mathfrak{r}}=\zeta_{\mathfrak{r}-1}+\zeta_{\mathfrak{r}}+k^{*} \delta \mid 1 \leq i \leq \mathfrak{r}-1\right\}$.

(ii)-(iv) are similarly proved.

Theorem 2.22. Suppose that $\Pi$ is a base of $R$ and $\alpha \in \Pi$ is a long root. Set $B:=\Pi \backslash\{\alpha\}$ and assume $W_{B}$ is the subgroup of the quasi-Weyl group $W$ of $R$ generated by the quasi-reflections based on the elements of $B$. Then $(\alpha, B)_{*} \neq\{0\}$ and for $S:=W_{B} B,\left(\operatorname{span}_{\mathbb{R}} S,(\cdot, \cdot)_{*}, S\right)$ is an irreducible finite root system with base $B$, either of type $D_{\ell}(\ell \geq 3)$ or $C_{\ell}(\ell \geq 2)$.

Proof. We carry out the proof through the following steps:

Step 1. $\mathbb{Z} \delta \cap \operatorname{span}_{\mathbb{R}} S=\mathbb{Z} \delta \cap \operatorname{span}_{\mathbb{R}} B=\{0\}$ : To the contrary, assume $\mathbb{Z} \delta \cap \operatorname{span}_{\mathbb{R}} B$ contains a nonzero element. Then there is a nonzero integer $k$ such that $k \delta \in$ $\operatorname{span}_{\mathbb{R}} B$. But

$$
2 k \delta \in R \subseteq \operatorname{span}_{\mathbb{Z} \geq 0}(B \cup\{\alpha\}) \cup \operatorname{span}_{\mathbb{Z} \leq 0}(B \cup\{\alpha\}) .
$$

Since $k \delta \in \operatorname{span}_{\mathbb{R}} B, 2 k \delta$ is a nonzero element of $R$ belonging to $\operatorname{span}_{\mathbb{Z} \geq 0} B \cup$ $\operatorname{span}_{\mathbb{Z} \leq 0} B$. Therefore, we have one of the following:

- $0 \neq 2 k \delta \in \operatorname{span}_{\mathbb{Z} \geq 0} B$ : In this case, $\alpha-2 k \delta \in R$ while it is an element of $\operatorname{span}_{\mathbb{Z}>0}\{\alpha\} \cup \operatorname{span}_{\mathbb{Z} \leq 0}(\Pi \backslash\{\alpha\})$ which is a contradiction.

- $0 \neq 2 k \delta \in \operatorname{span}_{\mathbb{Z} \leq 0} B$ : In this case, $\alpha+2 k \delta \in R$ while it is an element of $\operatorname{span}_{\mathbb{Z}>0}\{\alpha\} \cup \operatorname{span}_{\mathbb{Z} \leq 0}(\Pi \backslash\{\alpha\})$; again it is a contradiction.

Step 2. If $\alpha, \beta \in S$ with $(\alpha-\beta, B)_{*}=\{0\}$, then $\alpha=\beta$ : To the contrary, assume there are $\alpha, \beta \in S$ such that $\alpha \neq \beta$ and $(\alpha-\beta, B)_{*}=\{0\}$. Since $\operatorname{span}_{\mathbb{R}} B=\operatorname{span}_{\mathbb{R}} S$,

$$
(\alpha-\beta, B)_{*}=\{0\} \text { if and only if }(\alpha-\beta, S)_{*}=\{0\} .
$$

Case 1. $\alpha, \beta \in\left\{ \pm 2 \epsilon_{i}, \pm 2 \delta_{p} \mid 1 \leq i \leq m, 1 \leq p \leq n\right\}+\mathbb{Z} \delta$ : In this case, there are $p, q \in \mathbb{Z}$, elements $\zeta_{1}, \zeta_{2}$ of $\left\{\epsilon_{i}, \delta_{p} \mid 1 \leq i \leq m, 1 \leq p \leq n\right\}$ and $r_{1}, r_{2} \in\{ \pm 1\}$ such that $\alpha=2 r_{1} \zeta_{1}+p \delta$ and $\beta=2 r_{2} \zeta_{2}+q \delta$. Since $\alpha \in S,(\alpha-\beta, \alpha)_{*}=0$. This in turn implies that $\zeta_{1}=\zeta_{2}$ and $r_{1}-r_{2}=0$, i.e., $(p-q) \delta=\alpha-\beta \in \operatorname{span}_{\mathbb{R}} S=\operatorname{span}_{\mathbb{R}} B$. Using Step 1, we get that $p=q$. It means that $\alpha=\beta$.

Case 2. $\alpha \in\left\{ \pm \epsilon_{i} \pm \epsilon_{j}, \pm \delta_{p} \pm \delta_{q}, \pm \epsilon_{i} \pm \delta_{p} \mid 1 \leq i \neq j \leq m, 1 \leq p \neq q \leq n\right\}+\mathbb{Z} \delta$ and $\beta \in\left\{2 \epsilon_{i}, 2 \delta_{p} \mid 1 \leq i \leq m, 1 \leq p \leq n\right\}+\mathbb{Z} \delta$ : In this case, there are $p, q \in \mathbb{Z}$, $\zeta_{1}, \zeta_{2}, \zeta_{3} \in\left\{\epsilon_{i}, \delta_{p} \mid 1 \leq i \leq m, 1 \leq p \leq n\right\}$, with $\zeta_{1} \neq \zeta_{2}$, and $r_{1}, r_{2}, r_{3} \in\{ \pm 1\}$ such that $\alpha=r_{1} \zeta_{1}+r_{2} \zeta_{2}+p \delta$ and $\beta=2 r_{3} \zeta_{3}+q \delta$. If $\zeta_{3} \notin\left\{\zeta_{1}, \zeta_{2}\right\}$, then $0=(\alpha-\beta, \beta)_{*}=$ -4 which is a contradiction. So $\zeta_{3} \in\left\{\zeta_{1}, \zeta_{2}\right\}$, say e.g., $\zeta_{3}=\zeta_{1}$. So

$0=(\alpha-\beta, \beta)_{*}=\left(\left(r_{1}-2 r_{3}\right) \zeta_{3}+r_{2} \zeta_{2}+(p-q) \delta, 2 r_{3} \zeta_{3}+q \delta\right)_{*}=2 r_{3}\left(r_{1}-2 r_{3}\right) \neq 0$, a contradiction.

Case 3. $\alpha, \beta \in\left\{ \pm \epsilon_{i} \pm \epsilon_{j}, \pm \delta_{p} \pm \delta_{q}, \pm \epsilon_{i} \pm \delta_{p} \mid 1 \leq i \neq j \leq m, 1 \leq p \neq q \leq n\right\}+\mathbb{Z} \delta$ : Suppose that

$$
\alpha=r_{1} \zeta_{1}+r_{2} \zeta_{2}+p \delta \quad \text { and } \quad \beta=-r_{3} \zeta_{3}-r_{4} \zeta_{4}+q \delta,
$$


for some $p, q \in \mathbb{Z}$, elements $\zeta_{1}, \ldots, \zeta_{4}$ of $\left\{\epsilon_{i}, \delta_{j} \mid 1 \leq i \leq m, 1 \leq p \leq n\right\}$ with $\zeta_{1} \neq \zeta_{2}$ and $\zeta_{3} \neq \zeta_{4}$ and $r_{1}, \ldots, r_{4} \in\{ \pm 1\}$. We first assume $\left\{\zeta_{1}, \zeta_{2}\right\} \cap\left\{\zeta_{3}, \zeta_{4}\right\}=\emptyset$. Since $\zeta_{1} \in \operatorname{supp}(\alpha)$ and $\alpha \in S=W_{B} B$, we pick $\gamma \in B$ with $\zeta_{1} \in \operatorname{supp}(\gamma)$. Since $(\alpha-\beta, \gamma)_{*}=0$, there are $r, s \in\{ \pm 1\}, k \in \mathbb{Z}$ and $2 \leq i \leq 4$ with $r s=-r_{1} r_{i}$ and $\gamma=r \zeta_{1}+s \zeta_{i}+k \delta$. Since

$$
\eta:=r_{2} \zeta_{2}+r_{i} \zeta_{i}+\left(p-r r_{1} k\right) \delta=r_{\zeta_{1}+r s \zeta_{i}+r k \delta}\left(r_{1} \zeta_{1}+r_{2} \zeta_{2}+p \delta\right) \in W_{B} S \subseteq S,
$$

we have $2=(\eta, \alpha-\beta)_{*} \in(S, \alpha-\beta)_{*}=\{0\}$, a contradiction. So $\left\{\zeta_{1}, \zeta_{2}\right\} \cap\left\{\zeta_{3}, \zeta_{4}\right\} \neq$ $\emptyset$, say $\zeta_{1}=\zeta_{3}$.

If $r_{1}+r_{3} \neq 0$, then $r_{1}=r_{3}$. So $\alpha-\beta=2 r_{1} \zeta_{1}+r_{2} \zeta_{2}+r_{4} \zeta_{4}+(p-q) \delta$. As above, we find $\gamma \in B$ with $\zeta_{1} \in \operatorname{supp}(\gamma)$, so we have $(\gamma, \alpha-\beta) \neq 0$ which contradicts $(B, \alpha-\beta)_{*}=\{0\}$. So $r_{1}+r_{3}=0$. Therefore, we have

$$
\alpha-\beta=r_{2} \zeta_{2}+r_{4} \zeta_{4}+(p-q) \delta .
$$

Since $\zeta_{2} \in \operatorname{supp}(\alpha)$ and $\alpha \in S=W_{B} B$, we pick $\gamma \in B$ with $\zeta_{2} \in \operatorname{supp}(\gamma)$. One knows $(\gamma, \alpha-\beta)=\{0\}$, so if $\zeta_{2} \neq \zeta_{4}$, there are $k \in \mathbb{Z}$ and $r, s \in\{ \pm 1\}$ with $r s=-r_{2} r_{4}$ and $\gamma=r \zeta_{2}+s \zeta_{4}+k \delta$. But

$$
\eta:=r_{1} \zeta_{1}+r_{4} \zeta_{4}+\left(p-r_{2} r k\right) \delta=r_{\zeta_{2}+r s \zeta_{4}+r k \delta}\left(r_{1} \zeta_{1}+r_{2} \zeta_{2}+p \delta\right) \in S
$$

and $0=(\eta, \alpha-\beta)_{*}=1$, a contradiction, i.e., $\zeta_{2}=\zeta_{4}$ and so

$$
\alpha-\beta=\left(r_{2}+r_{4}\right) \zeta_{2}+(p-q) \delta .
$$

But $\zeta_{2}$ belongs to the support of an element $\theta \in B$. Since $(\alpha-\beta, \theta)_{*}=0$, we get that $r_{2}+r_{4}=0$. So using Step 1 , we get that $p-q=0$ and so $\alpha=\beta$.

Step 3. $S$ is a finite root system: Suppose $B=\left\{\beta_{1}, \ldots, \beta_{\ell}\right\}$ and define

$$
\begin{aligned}
\varphi: S & \longrightarrow \mathbb{Z}^{\ell} \\
\beta & \mapsto\left(\frac{2\left(\beta, \beta_{1}\right)_{*}}{\left(\beta_{1}, \beta_{1}\right)_{*}}, \ldots, \frac{2\left(\beta, \alpha_{\ell}\right)_{*}}{\left(\beta_{\ell}, \beta_{\ell}\right)_{*}}\right)
\end{aligned}
$$

We get using Step 2 that the map $\varphi$ is one to one. Moreover, for $1 \leq i \leq \ell$, $\frac{2\left(\beta, \beta_{i}\right)_{*}}{\left(\beta_{i}, \beta_{i}\right)_{*}} \in\{0, \pm 1, \pm 2\}$. Therefore, $S$ is finite; in particular, $\left(\operatorname{span}_{\mathbb{R}} S,(\cdot, \cdot)_{*}, S\right)$ is a finite root system.

Since $S$ is a finite root system, it is a direct sum of irreducible finite root systems; say $S=S_{1} \oplus \cdots \oplus S_{k}$. Setting $B_{i}:=B \cap S_{i}(1 \leq i \leq k)$, we have $S_{i}=W_{B_{i}} B_{i}$.

Step 4. There is a unique $i \in\{1, \ldots, k\}$ with $\operatorname{supp}\left(B_{i}\right) \cap \operatorname{supp}(\alpha) \neq \emptyset:$ Suppose $\operatorname{supp}(\alpha)=\{\epsilon\}$ and $\alpha=2 \operatorname{sgn}(\epsilon ; \alpha) \epsilon+k \delta$ for some $k \in \mathbb{Z}$. If $\epsilon \notin \operatorname{supp}(B)$, then for each $\eta \in \operatorname{supp}(B) \backslash\{\epsilon\}$,

$$
\epsilon+\eta \notin \mathbb{Z}(2 \operatorname{sgn}(\epsilon ; \alpha) \epsilon+k \delta)+\operatorname{span}_{\mathbb{Z}} B=\operatorname{span}_{\mathbb{Z}} \Pi
$$

which is a contradiction. Therefore, $\epsilon \in \operatorname{supp}(B)$ and so there is $1 \leq i \leq k$ with $\operatorname{supp}\left(B_{i}\right) \cap \operatorname{supp}(\alpha) \neq \emptyset$.

Now to the contrary, assume there is $1 \leq j \leq k$ with $i \neq j$ such that $\operatorname{supp}\left(B_{i}\right) \cap$ $\operatorname{supp}\left(B_{j}\right) \cap \operatorname{supp}(\alpha) \neq \emptyset$. So there are $\beta \in B_{i}$ and $\gamma \in B_{j}$ such that $\epsilon \in \operatorname{supp}(\beta) \cap$ $\operatorname{supp}(\gamma)$. Since $(\beta, \gamma)_{*}=0$, Fact 1 implies that $\operatorname{supp}(\beta)=\operatorname{supp}(\gamma)$ and $|\operatorname{supp}(\beta)|=$ $|\operatorname{supp}(\gamma)|=2$. Suppose $\operatorname{supp}(\beta)=\operatorname{supp}(\gamma)=\{\epsilon, \eta\}$. Since $\beta-\alpha, \gamma-\alpha \notin R$, Facts 1,2 imply that

$$
\operatorname{sgn}(\epsilon ; \beta)=-\operatorname{sgn}(\epsilon ; \alpha)=\operatorname{sgn}(\epsilon ; \gamma) \quad \text { and } \quad \operatorname{sgn}(\eta ; \beta)=-\operatorname{sgn}(\eta ; \alpha) .
$$


Also using Lemma 2.13(b)(i),(b)(iii), we have

$$
\operatorname{supp}(\theta) \cap\{\epsilon, \eta\}=\emptyset \quad(\theta \in \Pi \backslash\{\alpha, \beta, \gamma\}) .
$$

We recall that $(n, m) \neq(1,1)$ and choose $\zeta \in \operatorname{supp}(\Pi) \backslash\{\epsilon, \eta\}$, then

$$
\zeta+\operatorname{sgn}(\epsilon ; \alpha) \epsilon=r \alpha+s \beta+t \gamma+\sum_{\theta \in \Pi \backslash\{\alpha, \beta, \gamma\}} r_{\theta} \theta
$$

for some integers $r, s, t, r_{\theta}(\theta \in \Pi \backslash\{\alpha, \beta, \gamma\})$. This together with (2.13) and (2.14) implies that $1=2 r-s-t$ and $s=t$ which is a contradiction.

\section{Using Step 4, without loss of generality, we assume $\left(\alpha, B_{1}\right)_{*} \neq\{0\}$.}

Step 5. $B_{1}$ is a base of $S_{1}$ : We must prove that $S_{1} \subseteq \operatorname{span}_{\mathbb{Z} \geq 0} B_{1} \cup \operatorname{span}_{\mathbb{Z} \leq 0} B_{1}$. Suppose $\beta \in S_{1}$. We know that $R \subseteq \operatorname{span}_{\mathbb{Z} \geq 0} \Pi \cup \operatorname{span}_{\mathbb{Z} \leq 0} \Pi$. I $\beta \in R$, since $\beta \in \operatorname{span}_{\mathbb{Z}} B_{1}$, we get that $\beta \in \operatorname{span}_{\mathbb{Z} \geq 0} B_{1} \cup \operatorname{span}_{\mathbb{Z} \leq 0} B_{1}$ and so we are done. Next assume $\beta \in S_{1} \backslash R$. Then $\beta \in\left\{ \pm 2 \epsilon_{i}, \pm 2 \delta_{p} \mid 1 \leq i \leq m, 1 \leq p \leq n\right\}+\mathbb{Z} \delta$. The fact that $\left(\alpha, B_{1}\right) \neq\{0\}$ together with Lemma 2.10 implies that $S_{1}$ is not of simply-laced type. So there is a long root $\gamma \in B_{1}$. Assume

$$
\operatorname{supp}(\alpha)=\{\epsilon\}, \operatorname{supp}(\gamma)=\{\zeta\} \text { and } \operatorname{supp}(\beta)=\{\xi\} .
$$

Since $\left(\alpha, B_{1}\right)_{*} \neq\{0\}$, by Lemma 2.10 there is $\theta \in B_{1}$ with $|\operatorname{supp}(\theta)|=2$ and $\epsilon \in \operatorname{supp}(\theta)$. Also as $B_{1}$ is irreducible, again using Lemma 2.10, there is $\theta^{\prime} \in B_{1}$ with $\left|\operatorname{supp}\left(\theta^{\prime}\right)\right|=2$ and $\zeta \in \operatorname{supp}(\theta)$. Moreover, if $\xi \neq \zeta, \epsilon$, since $\beta \in S_{1}=W_{B_{1}} B_{1}$, there is $\theta^{\prime \prime} \in B_{1} \backslash\{\alpha, \gamma\}$ with $\left|\operatorname{supp}\left(\theta^{\prime \prime}\right)\right|=2$ and $\xi \in \operatorname{supp}\left(\theta^{\prime \prime}\right)$. These altogether imply that there is $t \in\{ \pm 1\}$ and $\rho \in\left(\left\{\epsilon_{i}, \delta_{p} \mid 1 \leq i \leq m, 1 \leq p \leq n\right\} \backslash\{\xi\}\right)+\mathbb{Z} \delta$ with $t \xi+\rho \in B_{1}$. Suuppose

$$
\Pi=\left\{\alpha_{1}, \ldots, \alpha_{\ell+1}\right\} \quad \text { with } \quad \alpha_{1}=\alpha, \alpha_{2}=\gamma, \alpha_{3}:=t \xi+\rho
$$

and

$$
B_{1}=\left\{\alpha_{2}, \ldots, \alpha_{\ell^{\prime}}\right\}
$$

Without loss of generality we assume

$$
t=\operatorname{sgn}(\xi ; \beta) .
$$

Again using Lemma 2.10, we may assume $\epsilon, \xi \in\left\{\epsilon_{i} \mid 1 \leq i \leq m\right\}$ and $\zeta \in\left\{\delta_{p} \mid 1 \leq\right.$ $p \leq n\}$ or $\epsilon, \xi \in\left\{\delta_{p} \mid 1 \leq p \leq n\right\}$ and $\zeta \in\left\{\epsilon_{i} \mid 1 \leq i \leq m\right\}$. So since $\beta \notin R$, there are $r \in\{ \pm 1\}, k, k^{\prime} \in \mathbb{Z}$ and $s \in\{0,1\}$ with

$$
\beta=2 t \xi+2 k \delta+s \delta \quad \text { and } \quad \gamma=2 r \zeta+2 k^{\prime} \delta+s \delta .
$$

Since $t \xi-\rho+2 k \delta+s \delta, t \xi-r \zeta+\left(k-k^{\prime}\right) \delta \in R$, there are $r_{i}, t_{i} \in \mathbb{Z}(1 \leq i \leq \ell+1)$ where $t_{i}$ 's (resp. $r_{i}$ 's) are all non-positive or all non-negative with

$$
t \xi-r \zeta+k \delta-k^{\prime} \delta=\sum_{i=1}^{\ell+1} t_{i} \alpha_{i} \quad \text { and } \quad t \xi-\rho+2 k \delta+s \delta=\sum_{i=1}^{\ell+1} r_{i} \alpha_{i}
$$

and we have

\footnotetext{
${ }^{3}$ We remind that quasi-reflections do not necessarily preserves $R$.
} 


$$
\begin{aligned}
\left(1+r_{3}\right) \alpha_{3}+\sum_{3 \neq i=1}^{\ell+1} r_{i} \alpha_{i} & =(t \xi-\rho+2 k \delta+s \delta)+(t \xi+\rho) \\
& =\underbrace{2 t \xi+2 k \delta+s \delta}_{\beta} \\
& =2\left(t \xi-r \zeta+k \delta-k^{\prime} \delta\right)+2 r \zeta+2 k^{\prime} \delta+s \delta \\
& =\left(1+2 t_{2}\right) \alpha_{2}+\sum_{2 \neq i=1}^{\ell+1} 2 t_{i} \alpha_{i} .
\end{aligned}
$$

Since $\beta \in S_{1} \subseteq \operatorname{span}_{\mathbb{Z}} B_{1}$, we get

$$
\left(1+r_{3}\right) \alpha_{3}+\sum_{3 \neq i=2}^{\ell^{\prime}} r_{i} \alpha_{i}=\beta=\left(1+2 t_{2}\right) \alpha_{2}+\sum_{i=3}^{\ell^{\prime}} 2 t_{i} \alpha_{i} .
$$

If $\beta \notin \operatorname{span}_{\mathbb{Z} \geq 0} B_{1} \cup \operatorname{span}_{\mathbb{Z} \leq 0} B_{1}$, using the same argument as in the proof of Proposition [2.6] we get $r_{3}=0$ and so $1=2 t_{3}$, a contradiction.

Step 6. $k=1$ : Suppose $k \geq 2$. Recall that $S_{1}$ is an irreducible finite root system with base $B_{1}$ and that $\{0\} \neq\left(\alpha_{1}, B_{1}\right)_{*} \subseteq\left(\alpha_{1}, S_{1}\right)_{*}$. Using Lemma 2.19, we have the following cases:

Case 1. $S_{1}$ is of type $D_{\mathfrak{r}}(\mathfrak{r} \geq 4)$ : By Corollary 2.21(i) and Step 5, there are

$$
r \in\{0,1\}, \quad k^{*} \in \mathbb{Z} \quad \text { and } \zeta_{1}, \ldots, \zeta_{\mathfrak{r}} \in\left\{ \pm \epsilon_{\mathfrak{r}}, \pm \delta_{p} \mid 1 \leq t \leq n, 1 \leq p \leq m\right\}+\mathbb{Z} \delta
$$

with $\operatorname{supp}\left(\zeta_{i}\right)=\left\{\dot{\zeta}_{i}\right\}$ such that $\operatorname{supp}(\alpha)=\left\{\dot{\zeta}_{1}\right\}, \dot{\zeta}_{i}$ 's are distinct,

$\alpha=-2 \zeta_{1}+r \delta \quad$ and $\quad B_{1}=\left\{\gamma_{i}:=\zeta_{i}-\zeta_{i+1}, \gamma_{\mathfrak{r}}:=\zeta_{\mathfrak{r}-1}+\zeta_{\mathfrak{r}}+k^{*} \delta \mid 1 \leq i \leq \mathfrak{r}-1\right\}$.

Now we have

$$
\left(r+k^{*}\right) \delta=\gamma+2 \alpha_{1}+\cdots+2 \gamma_{\mathfrak{r}-2}+\alpha_{\mathfrak{r}-1}+\alpha_{\mathfrak{r}} .
$$

Since $\left\{\alpha, \gamma_{1}, \ldots, \gamma_{\mathfrak{r}}\right\}$ is linearly independent, $k^{*}+r \neq 0$, so $\delta \in \operatorname{span}_{\mathbb{Z}} B_{1} \cup\{\alpha\}$. Next fix a root $\rho \in B_{2}$. If $\delta \in \operatorname{span}_{\mathbb{Z} \geq 0} B_{1} \cup\{\alpha\}$, then $-\rho+2 \delta$ is an element of $R \cap\left(\operatorname{span}_{\mathbb{Z}<0} B_{2}+\operatorname{span}_{\mathbb{Z} \geq 0} B_{1} \cup\{\alpha\}\right)$ which is a contradiction. Similarly, if $\delta \in$ $\operatorname{span}_{\mathbb{Z} \leq 0} B_{1} \cup\{\alpha\}$, we have $\rho+2 \delta \in R \cap\left(\operatorname{span}_{\mathbb{Z}>0} B_{2}+\operatorname{span}_{\mathbb{Z} \leq 0} B_{1} \cup\{\alpha\}\right)$ which is again a contradiction.

Case 2. $S_{1}$ is of type $C_{\mathfrak{r}}(\mathfrak{r} \geq 2)$. We get a contradiction using the same argument as in Case 1.

Case 3. $S_{1}$ is of type $A_{\mathfrak{r}}(\mathfrak{r} \neq 3)$. By Corollary 2.21(iii) and Step 5, there are

$$
r \in\{0,1\}, \zeta_{1}, \ldots, \zeta_{\mathfrak{r}+1} \in\left\{ \pm \epsilon_{t}, \pm \delta_{p} \mid 1 \leq t \leq n, 1 \leq p \leq m\right\}+\mathbb{Z} \delta
$$

with $\operatorname{supp}\left(\zeta_{i}\right)=\left\{\dot{\zeta}_{i}\right\}$ such that $\operatorname{supp}(\alpha)=\left\{\dot{\zeta}_{1}\right\}, \dot{\zeta}_{i}$ 's are distinct,

$$
\alpha=-2 \zeta_{1}+r \delta \quad \text { and } \quad B_{1}=\left\{\alpha_{i}:=\zeta_{i}-\zeta_{i+1} \mid 1 \leq i \leq \mathfrak{r}\right\} .
$$

If $2 \leq j \leq \mathfrak{r}+1$ and $\dot{\zeta}_{j} \in \operatorname{supp}\left(B_{i}\right)$ for some $i \neq 1$, then there is $\beta \in B_{i}$ with $\dot{\zeta}_{j} \in \operatorname{supp}(\beta)$. Since $\left(B_{1}, B_{i}\right)_{*}=\{0\}$, we have $\left(\alpha_{j-1}, \beta\right)_{*}=\left(\alpha_{j}, \beta\right)_{*}=0$ and so by Fact 1 , we have $\operatorname{supp}\left(\alpha_{j-1}\right)=\operatorname{supp}(\beta)=\operatorname{supp}\left(\alpha_{j}\right)$ which is a contradiction. Therefore, contemplating Step 4. we have

$$
\operatorname{supp}\left(B_{1}\right) \cap \cup_{i=2}^{k} \operatorname{supp}\left(B_{i}\right)=\emptyset .
$$


But there are $r_{1}, \ldots, r_{\mathfrak{r}+1} \in \mathbb{Z}$ with

$$
\delta=r_{1}\left(-2 \zeta_{1}+r \delta\right)+\sum_{i=2}^{\mathfrak{r}+1} r_{i}\left(\zeta_{i-1}-\zeta_{i}\right)+\sum_{\alpha \in \cup_{i \geq 2} B_{i}} k_{\alpha} \alpha
$$

So using (2.15), $2 r_{1}=r_{2}=\cdots=r_{\mathfrak{r}+1}=0$, in other words, $\delta \in \operatorname{span}_{\mathbb{Z}} \cup_{i=2}^{k} B_{i}$ which is a contradiction as the form $(\cdot, \cdot)_{*}$ is nondegenerate on $\operatorname{span}_{\mathbb{R}} S$.

Case 4. $S_{1}$ is of type $A_{3}$ : Using Corollary 2.21(iv) together with the same argument as in Cases 1 and 3 , we get a contradiction. This completes the proof.

Step 7. If $\ell \neq 3$, then $S$ is not of type $A$ : Since $S=S_{1}$ is of rank $\ell=m+n$, if it is of type $A$, by Corollary 2.21(iii) and Step 5 , there are $\ell+1$ elements $\zeta_{1}, \ldots, \zeta_{\ell+1}$ of $\left\{ \pm \epsilon_{i}, \pm \delta_{p} \mid 1 \leq i \leq m, 1 \leq p \leq n\right\}$ with $\zeta_{i} \neq \pm \zeta_{j}$ if $i \neq j$ which is a contradiction.

Steps 3,5,6 and 7 together with Lemma 2.19 complete the proof.

Lemma 2.23. Suppose that $B$ is a base of $R$ without long roots, then $B$ is of the form $\pm B^{1}$. Set

$$
\xi_{i}:= \begin{cases}\delta_{i} & 1 \leq i \leq n \\ \epsilon_{i-n} & n+1 \leq i \leq \ell\end{cases}
$$

and

$$
\Pi:=\left\{\delta-\left(\xi_{1}+\xi_{2}\right), \xi_{i}-\xi_{i+1}, \xi_{\ell-1}+\xi_{\ell} \mid 1 \leq i \leq \ell-1\right\} .
$$

Then $\Pi$ is a base of $R$ and each base of $R$ which does not contain long roots is conjugate with $\pm \Pi$ under quasi-Weyl group.

Proof. That $\Pi$ is a base of $R$ follows from Proposition 2.9. Suppose that $B$ is a base of $R$ which does not contain long roots. Set

$$
\begin{aligned}
S: & = \pm\left\{0, \epsilon_{i} \pm \epsilon_{j}, \delta_{p} \pm \delta_{q}, \epsilon_{i} \pm \delta_{p} \mid 1 \leq i \neq j \leq m, 1 \leq p \neq q \leq n\right\}+\mathbb{Z} \delta \\
& =\left\{0, \pm \xi_{i} \pm \xi_{j} \mid 1 \leq i \neq j \leq \ell\right\}+\mathbb{Z} \delta .
\end{aligned}
$$

Then $\left(\operatorname{span}_{\mathbb{R}} S,(\cdot, \cdot)_{*}, S\right)$ is the root system of $D_{\ell}^{(1)}$ and $\Pi$ is a base of $R$. Since $B$ is a subset of $S$, it is a base of $S$. So there is an element $\omega$ of the Weyl group of $S$ such that $\omega(\Pi)=r B$ for some $r \in\{ \pm 1\}$. Setting

$$
\theta_{i}:=r \omega\left(\xi_{i}\right) \in\left\{ \pm \xi_{j} \mid 1 \leq j \leq \ell\right\}+\mathbb{Z} \delta \quad(1 \leq i \leq \ell)
$$

we have

$$
B=\left\{r \delta-\left(\theta_{1}+\theta_{2}\right), \theta_{i}-\theta_{i+1}, \theta_{\ell-1}+\theta_{\ell}\right\}
$$

Since

$$
2 \theta_{1}-r \delta=\left(\theta_{1}-\theta_{2}\right)-\left(r \delta-\left(\theta_{1}+\theta_{2}\right)\right), 2 \theta_{\ell}=\left(\theta_{\ell-1}+\theta_{\ell}\right)-\left(\theta_{\ell-1}-\theta_{\ell}\right) \in B-B,
$$

we have

$$
2 \theta_{1}-r \delta, 2 \theta_{\ell} \notin R
$$

and so

$$
\operatorname{supp}\left(\theta_{1}\right) \subseteq\left\{\delta_{p} \mid 1 \leq p \leq n\right\} \quad \text { and } \operatorname{supp}\left(\theta_{\ell}\right) \subseteq\left\{\epsilon_{i} \mid 1 \leq i \leq m\right\} .
$$

This completes the proof as the Weyl group of $S$ is a subgroup of the quasi-Weyl group of $R$.

Proposition 2.24. $\quad$ (i) If $\Pi$ is a base of $R$ without long roots, then $\Pi$ is of the form $\pm B^{1}$. 
(ii) If $\Pi$ is a base of $R$ having a unique long root which belongs to $\left\{ \pm 2 \delta_{p} \mid 1 \leq\right.$ $p \leq n\}+2 \mathbb{Z} \delta$, then $\Pi$ is of the form $\pm B^{2}$.

(iii) If $\Pi$ is a base of $R$ having a unique long root which belongs to $\left\{ \pm 2 \epsilon_{i} \mid 1 \leq\right.$ $i \leq m\}+2 \mathbb{Z} \delta+\delta$, then it is of the form $\pm B^{3}$.

(iv) If $\Pi$ is a base of $R$ having two long roots, then it is of the form $\pm B^{4}$.

Proof. (i) See Lemma 2.23

(ii) Using Theorem 2.22 and Corollary 2.21(i),(iv), we find $k^{*} \in \mathbb{Z}$ and $\theta_{1}, \ldots, \theta_{\ell} \in$ $\left\{ \pm \epsilon_{t}, \pm \delta_{p} \mid 1 \leq t \leq m, 1 \leq p \leq n\right\}+\mathbb{Z} \delta$ with $\operatorname{supp}\left(\theta_{i}\right)=\left\{v_{i}\right\}$ such that $v_{i}$ 's are distinct and

$$
\Pi=\left\{-2 \theta_{1}, \theta_{i}-\theta_{i+1}, \theta_{\ell-1}+\theta_{\ell}+k^{*} \delta \mid 1 \leq i \leq \ell-1\right\} .
$$

Since $\delta \in \operatorname{span}_{\mathbb{Z}} \Pi$, it follows that $k^{*}= \pm 1$. Also as

$$
2 \theta_{\ell}+k^{*} \delta=\left(\theta_{\ell-1}+\theta_{\ell}+k^{*} \delta\right)-\left(\theta_{\ell-1}-\theta_{\ell}\right) \in \Pi-\Pi,
$$

we get that $2 \theta_{\ell}+k^{*} \delta \notin R$ and so $v_{\ell} \in\left\{\delta_{p} \mid 1 \leq p \leq n\right\}$.

(iii) Using Theorem 2.22 and Corollary 2.21(i),(iv), we find $k^{*} \in \mathbb{Z}$ and $\theta_{1}, \ldots, \theta_{\mathfrak{r}} \in$ $\left\{ \pm \epsilon_{t}, \pm \delta_{p} \mid 1 \leq t \leq n, 1 \leq p \leq m\right\}+\mathbb{Z} \delta$ with $\operatorname{supp}\left(\theta_{i}\right)=\left\{v_{i}\right\}$ such that $v_{i}$ 's are distinct and

$$
\Pi=\left\{-2 \theta_{1}+\delta, \theta_{i}-\theta_{i+1}, \theta_{\ell-1}+\theta_{\ell}+k^{*} \delta \mid 1 \leq i \leq \ell-1\right\} .
$$

Since $\delta \in \operatorname{span}_{\mathbb{Z}} \Pi$, it follows that $k^{*} \in\{0,-2\}$. Also as

$$
2 \theta_{\ell}+k^{*} \delta=\left(\theta_{\ell-1}+\theta_{\ell}+k^{*} \delta\right)-\left(\theta_{\ell-1}-\theta_{\ell}\right) \in \Pi-\Pi,
$$

we get that $2 \theta_{\ell}+k^{*} \delta \notin R$ and so $v_{\ell} \in\left\{\epsilon_{i} \mid 1 \leq i \leq m\right\}$. Therefore, if $k^{*}=0$, we are done. But if $k^{*}=-2$, setting $\theta_{i}^{\prime}:=\theta_{i}-\delta(1 \leq i \leq \ell)$, we have

$$
\Pi=\left\{-2 \theta_{1}^{\prime}-\delta, \theta_{i}^{\prime}-\theta_{i+1}^{\prime}, \theta_{\ell-1}^{\prime}+\theta_{\ell}^{\prime} \mid 1 \leq i \leq \ell-1\right\}
$$

which is of the form $-B^{3}$.

(iv) Using Theorem 2.22 and Corollary 2.21)(ii), we find $k^{*} \in \mathbb{Z}$ and $\theta_{1}, \ldots, \theta_{\mathfrak{r}} \in$ $\left\{ \pm \epsilon_{t}, \pm \delta_{p} \mid 1 \leq t \leq n, 1 \leq p \leq m\right\}+\mathbb{Z} \delta$ with $\operatorname{supp}\left(\theta_{i}\right)=\left\{v_{i}\right\}$ such that $v_{i}$ 's are distinct and

$$
\Pi=\left\{-2 \theta_{1}, \theta_{i}-\theta_{i+1}, 2 \theta_{\ell}+k^{*} \delta \mid 1 \leq i \leq \ell-1\right\} .
$$

Since $\delta \in \operatorname{span}_{\mathbb{Z}} \Pi$, it follows that $k^{*} \in\{ \pm 1\}$. This completes the proof.

Theorem 2.25. Suppose $R=A(2 m-1,2 n-1)^{(2)}$ for some positive integers $m, n$ with $(m, n) \neq(1,1)$. Then we have the following:

(i) Each base of $R$ is of the form $\pm B^{j}$ for some $1 \leq j \leq 4$.

(ii) $\mathrm{Set}$

$$
\xi_{i}:= \begin{cases}\delta_{i} & 1 \leq i \leq n \\ \epsilon_{i-n} & n+1 \leq i \leq \ell\end{cases}
$$

and

$$
\begin{aligned}
& \Pi^{1}:=\left\{\delta-\left(\xi_{1}+\xi_{2}\right), \xi_{i}-\xi_{i+1}, \xi_{\ell-1}+\xi_{\ell} \mid 1 \leq i \leq \ell-1\right\}, \\
& \Pi^{2}:=\left\{-2 \xi_{2}, \xi_{i}-\xi_{i+1}, \xi_{\ell}-\xi_{1}, \xi_{\ell}+\xi_{1}+\delta \mid 2 \leq i \leq \ell-1\right\} \quad(\text { if } n \geq 2), \\
& \Pi^{3}:=\left\{-2 \xi_{\ell}+\delta, \xi_{\ell}-\xi_{1}, \xi_{i}-\xi_{i+1}, \xi_{\ell-2}+\xi_{\ell-1} \mid 1 \leq i \leq \ell-2\right\} \quad(\text { if } m \geq 2), \\
& \Pi^{4}:=\left\{-2 \xi_{1}, \xi_{i}-\xi_{i+1}, 2 \xi_{\ell}+\delta \mid 1 \leq i \leq \ell-1\right\} .
\end{aligned}
$$

Then $\Pi^{1}, \ldots, \Pi^{4}$ are bases of $R$ and if $\Pi$ is a base of $R$, then there is $w \in W$ and $1 \leq i \leq 4$ such that $\Pi= \pm w\left(\Pi^{i}\right)$. 
(iii) Each base of $R$ is an $S$-base.

Proof. (i) See Proposition 2.24. 2.18 .

(ii) follows from part (i), Lemma 2.23 and Proposition 2.9 together with Corollary

(iii) Set

$$
\dot{R}:=\left\{0, \pm 2 \epsilon_{i}, \pm 2 \delta_{p}, \pm \epsilon_{i} \pm \epsilon_{j}, \pm \delta_{p} \pm \delta_{q}, \pm \epsilon_{i} \pm \delta_{p} \mid 1 \leq i \neq j \leq m, 1 \leq p \neq q \leq n\right\}
$$

and assume $\Pi$ is a base of $R$. Using part (i) together with Lemma 2.23 and Proposition 2.9 for each $\alpha \in \dot{R}$, there is a positive integer $N$ such that either

$$
R \cap\left(\alpha+\mathbb{Z}^{\geq N}\right) \subseteq R^{+}(\Pi) \text { and } R \cap\left(\alpha+\mathbb{Z}^{\leq N}\right) \subseteq R^{-}(\Pi)
$$

or

$$
R \cap\left(\alpha+\mathbb{Z}^{\leq N}\right) \subseteq R^{+}(\Pi) \text { and } \quad R \cap\left(\alpha+\mathbb{Z}^{\geq N}\right) \subseteq R^{-}(\Pi)
$$

This implies that if $\Pi$ and $\Pi^{\prime}$ are two bases of $R$, then either $R^{+}(\Pi) \cap R^{-}\left(\Pi^{\prime}\right)$ or $R^{+}(\Pi) \cap R^{+}\left(\Pi^{\prime}\right)$ is a finite set. In particular, if $\Pi$ is the standard base of $R$, for each base $\Pi^{\prime}$ of $R$, either $R^{+}(\Pi) \cap R^{-}\left(\Pi^{\prime}\right)$ or $R^{+}(\Pi) \cap R^{+}\left(\Pi^{\prime}\right)$ is a finite set. Now the result follows using Lemma 2.3 as the standard base is in fact an S-base.

\section{REFERENCES}

[1] M. Gorelik, and A. Shaviv, Generalized reflection root systems, J. Algebra 491 (2017), 490516.

[2] V. Kac, Lie superalgebras, Adv. Math. 26 (1977), 8-96.

[3] V. Kac, Infinite-dimensional Lie algebras. Third edition. Cambridge University Press, Cambridge, 1990.

[4] E. Neher, Extended affine Lie algebras and other generalization of affine Lie algebras- a survey, Developments and trends in infinite-dimensional Lie theory, 53-126, Prog. Math., 228, Birkhauser Boston, Inc., Boston, MA, (2011).

[5] V. Serganova, On generalizations of root systems, Comm. in Alg. 24 (13) (1996), 4281-4299.

[6] V. Serganova, Kac-Moody superalgebras and integrebility, Developments and Trends in Infinite-Dimensional Lie Theory, 169-218.

[7] J.W. Van de Lour, A classification of Contragrediant Lie superalgebras of finite growth, Comm. in Algebra, 17 (8) (1989) 1815-1841.

[8] J.W. Van de Lour, Contragredientlie superalgebras of finite growth, Ph. D. Thesis, Utrecht University, 1986.

[9] M. Yousofzadeh, Locally finite root supersystems, Comm. in Alg. 45 (10) (2017), 4292-4320.

[10] M. Yousofzadeh, Extended affine Lie superalgebras, Publ. Res. Inst. Math. Sci. 52 (3) (2016), no. 3, 309-333.

[11] M. Yousofzadeh, Extended affine root supersystems, J. Algebra 449 (2016), 539-564. 